\title{
Article \\ Geochemistry of Pyritic Mudstones from the Singa Formation, Malaysia: Insights into Gold Potential, Source of Sulfur and Organic Matter
}

\author{
Charles Makoundi ${ }^{1, *} \mathbb{C}$, Zakaria Endut ${ }^{2, *}$, Ross R. Large ${ }^{1}$, Khin Zaw ${ }^{1}$, Elena Lounejeva ${ }^{1}$, Mohd Shafeea Leman ${ }^{3,4}$, \\ Kamal Roslan Mohamed ${ }^{3,4}$ and Mohd Basril Iswadi Basori ${ }^{3}$ \\ 1 Centre for Ore Deposit and Earth Science, University of Tasmania, Private Bag 126, \\ Hobart, TAS 7001, Australia; ross.large@utas.edu.au (R.R.L.); KhinZaw.nilar@gmail.com (K.Z.); \\ elena.lounejeva@utas.edu.au (E.L.) \\ 2 School of Materials and Mineral Resources Engineering, Universiti Sains Malaysia, \\ Nibong Tebal 14300, Penang, Malaysia \\ 3 Geology Programme, School of Environment and Nature Resource Sciences, Faculty of Science \\ and Technology, Universiti Kebangsaan Malaysia, Bangi 43600, Selangor, Malaysia; \\ mshafeea@gmail.com (M.S.L.); kamal@ukm.my (K.R.M.); basril@ukm.edu.my (M.B.I.B.) \\ 4 Langkawi Research Centre, Institute for Environment and Development (Lestari), Jalan Teluk Yu, \\ Kampung Kok, Langkawi 07000, Kedah, Malaysia \\ * Correspondence: c.makoundi@utas.edu.au (C.M.); zakaria.endut@usm.my (Z.E.); \\ Tel.: +61-36-226-2472 (C.M.); +60-19-982-8334 (Z.E.)

Citation: Makoundi, C.; Endut, Z.; Large, R.R.; Zaw, K.; Lounejeva, E.; Leman, M.S.; Mohamed, K.R.; Basori, M.B.I. Geochemistry of Pyritic Mudstones from the Singa Formation, Malaysia: Insights into Gold

Potential, Source of Sulfur and Organic Matter. Geosciences 2021, 11, 279. https://doi.org/10.3390/ geosciences 11070279

Academic Editors: Marco Viccaro and Jesus Martinez-Frias

Received: 10 May 2021

Accepted: 29 June 2021

Published: 2 July 2021

Publisher's Note: MDPI stays neutra with regard to jurisdictional claims in published maps and institutional affiliations.

Copyright: (c) 2021 by the authors. Licensee MDPI, Basel, Switzerland. This article is an open access article distributed under the terms and conditions of the Creative Commons Attribution (CC BY) license (https:// creativecommons.org/licenses/by/ $4.0 /)$
Abstract: Major trace element analyses, including pyrite chemistry of pyritic mudstones of shallowmarine Singa Formation of Pennsylvanian-Early Permian age have been carried out to assess gold potential, the source of sulfur and organic matter. Regionally, Singa Formation spatially correlates with the Bohorok Formation (Sumatra, Indonesia), the Kaeng Krachang group (Thailand), and the Lebyin group (Burma or Myanmar). In Southeast Asia, this formation is important because it has a record of glacial processes that occurred along the northern margin of Gondwana in the Late Paleozoic age. This study has revealed that mudstones of the Singa Formation, which contain lonestones of glacial origin, deposited under suboxic-oxic conditions in shallow marine environment during Pennsylvanian-Early Permian time. The black mudstones contain total organic carbon which ranges from 0.1 to $0.7 \mathrm{wt} . \%$, and gold content varying from 40 to $62 \mathrm{ppb}$, making them gold source rocks. This study has revealed diagenetic gold presence in the early pyrite generations (pyrites 1, 2, and 3) in these mudstones with gold content ranging up to $1.6 \mathrm{ppm}$ Au which is indicative of early enrichment of gold. Conversely, late generations of pyrite (pyrites 4, 5, and 6) in these mudstones record low gold content up to $0.5 \mathrm{ppm} \mathrm{Au}$. The $\delta^{34} \mathrm{~S}$ values for pyrite grains range from $-24.6 \%$ to $+6.2 \%$ likely indicate a combination of magmatic and biogenic source of sulfur. Organic carbon isotope composition of the pebbly mudstone samples shows a wide range from $-23.9 \%$ to $-5.8 \%$ o indicating a mixed terrestrial and marine source.

Keywords: gold; source rock; X-ray fluorescence analysis; geochemistry; Malaysia

\section{Introduction}

Fine-grained sedimentary sequences host several trace elements, including $\mathrm{Zn}, \mathrm{Pb}, \mathrm{Cu}$, $\mathrm{Mo}, \mathrm{Se}, \mathrm{V}, \mathrm{U}, \mathrm{Ni}, \mathrm{As}, \mathrm{Ag}, \mathrm{Sb}, \mathrm{Cr}$, and Au [1-4]. Black shales (commonly metamorphosed to greenschist facies) are host to significant gold deposits in certain regions in the world such as the Chuniespoort Group of the Late Archean-Early Proterozoic Transvaal Sequence (South Africa), the Central Gold Belt (Malaysia), and the Sepon Mineral District (Laos), the Mathinna Turbidite deformed sequence in Northeast Tasmania (Australia), Upper Devonian black shale series of the Xikuangshan Sb deposit in Hunan (China) [5-9]. Paleozoic glacial deposits are cropping out in Southeast Asia [10]. 
This study focuses on the Late Carboniferous (Pennsylvanian)-Early Permian Singa Formation (Figure 1), which was deposited during Late Paleozoic glacial times [11] and prior to the Pangea break-up [12]. Argillaceous sequences including black or dark mudstones are distributed in the Langkawi Islands. These mudstones have never been investigated for their trace element content. It begs the question whether the black mudstones can be source rocks for metals in the district. The element of strong interest is the proximity of the Singa Formation to the locally exposed granitic outcrops (which is a possible source of magmatic fluids), and its connection with other formations across the region.
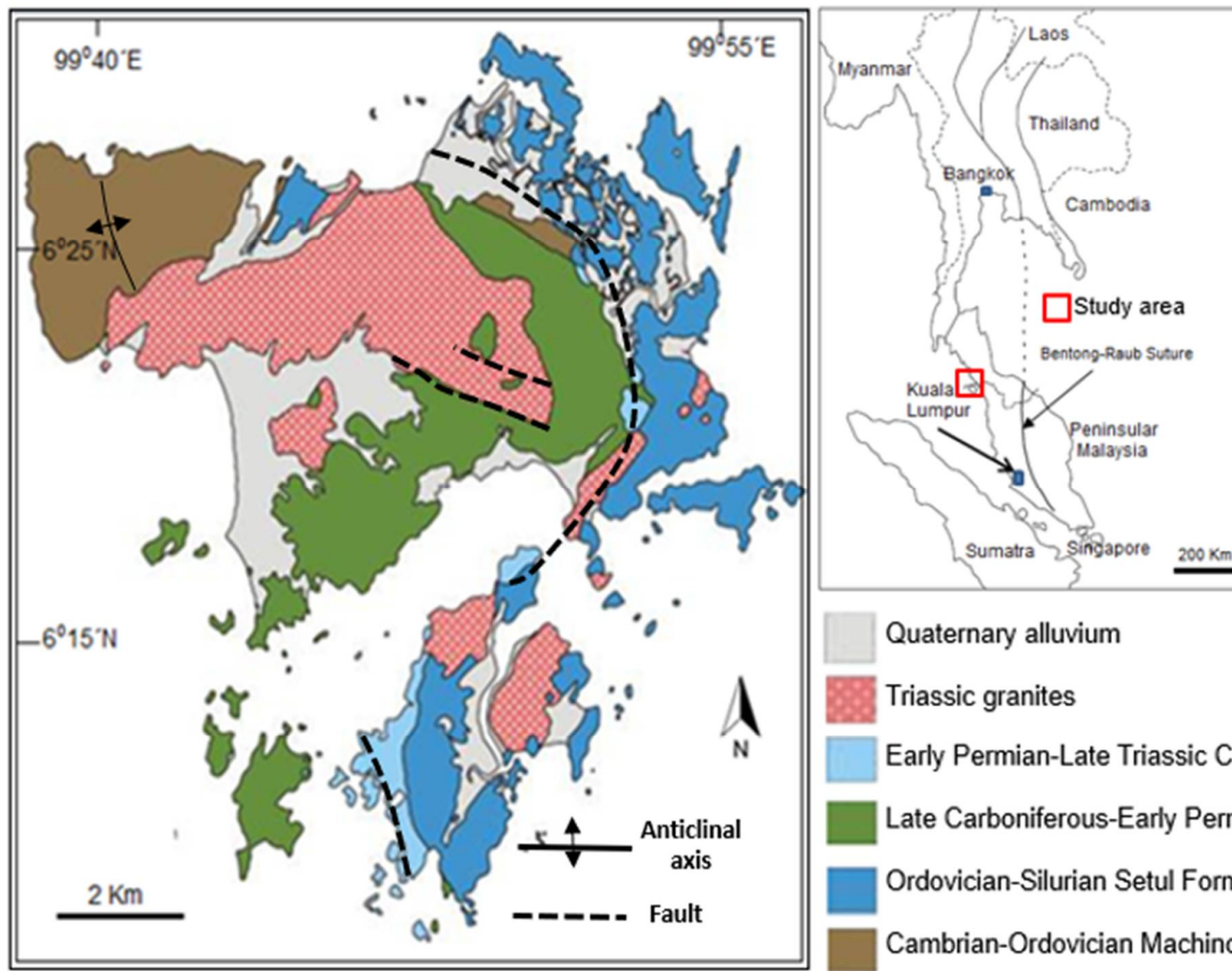

Quaternary alluvium

Triassic granites

Early Permian-Late Triassic Chuping Formation

Late Carboniferous-Early Permian Singa Formation

Ordovician-Silurian Setul Formation

Cambrian-Ordovician Machinchang Formation

Figure 1. Geological sketch map of Langkawi Islands, Malaysia (modified after Che et al., 2008) [11].

In this study, we report the metal content of black mudstones for the Singa Formation evaluated from both pyrite trace element chemistry and whole rock analyses. The significance of this study lies in understanding the redox conditions over the deposition of these mudstones in the Gondwanaland Sea and to also account for gold source rock potential. The reason being is that in many orogenic gold systems, mudstones or shales were found to contain early enrichment of gold in their first generation of sedimentary pyrite followed by later remobilization of gold to concentrate in structural traps during basin inversion or subsequent tectonic deformation. This study investigated whether sedimentary pyrites existing in the Singa mudstones contain gold and trace elements that would warrant further metallogenic research.

The Central Gold Belt of Peninsular Malaysia is known to contain mudstones associated with significant gold-enriched structures which have been in the very core of economic geology research, mineral exploration, and mining in Malaysia. In this study, the trace element content of these mudstones is compared to that of carbonaceous shales which are widely known to be best gold source rocks [1-4].

In addition, knowing the origin and isotopic signature of the organic carbon and sulfur is important as mudstones or shales in orogenic gold systems often have elevated 
content of organic carbon and sulfur reflecting the redox conditions (oxic to euxinic) of the ocean during their deposition. The source of organic carbon and sulfur of these black or dark mudstones is an important contribution in understanding the origin of organic matter and sulfur from these clast-bearing mudstones in the Sibumasu Terrane.

\section{Geological Setting}

Paleozoic sedimentary rocks and granite crop out on the Langkawi Islands [13]. The distribution of these rocks is shown in Figure 1. The oldest Paleozoic sequence is the Machinchang Formation, which consists mainly of three Members. The oldest Hulur Member, which is a coarsening upward sequence that contains interbedded graded siltstone, grey shale, and clayey sandstone, is interpreted as having been deposited in a prodelta environment. The Chinchin Member which consists of a fining upward sequence of quartzose conglomerate and sandstone, was deposited in upper shoreface to beach environment. The youngest Jemuruk Member made up of fining upward succession of siltstone, shale, hummocky cross-bedded sandstone, and thin beds of limestone, accumulated in storm-derived shoreface to back barrier lagoon with tidal channel environment. In the northwest domain, the Machinchang Formation is overlain by shallow marine limestone of the Setul Formation.

The Setul Formation comprises two Members including the Lower Detrital Band (LDB) and the Upper Limestone Member that represent transgressive sediment input onto the shelf limestone (Lower Ordovician) during sedimentation. The lower detrital member of the Setul Formation is made up of black siliceous argillite, chert, quartzite, and siltstone.

Unconformably, above the Setul Formation are dark grey argillaceous shales of the Singa formation which has a maximum thickness of $1500 \mathrm{~m}$ [14]. The top of the Singa Formation is marked by an unconformity implying a break in sedimentation. Above the unconformity is the $600 \mathrm{~m}$ thick Chuping Formation, cropping out on the south and east of Langkawi islands and composed of bedded, dark grey limestone with chert nodules at the base of the Chuping Formation [15] (Figure 1). Granitic exposures or suite of Triassic age is found intruding older rocks changing them to metamorphic rocks. The Rebanggun beds or red beds are found at the base of the Singa Formation. They consist of conglomeratic mudstones.

The Singa Formation crops out in the NW region (known as the Kubang Pasu Formation) of Peninsular Malaysia, which is an extended arm of the Malaysian Western Belt within the Sibumasu Terrane. The Western belt originated from the NW Australian Gondwana margin in the Late Early Permian [16]. The term Sibumasu is an acronym, which represents the combination of SI (Sino, Siam), BU (Burma, now Myanmar), MA (Malaya) and SU (Sumatra) [17].

\section{Singa Formation}

The Pennsylvanian-Early Permian Singa Formation exposures were present at the Tanjung Malie beach showing interbedded massive sandstone, greywacke, and black or dark pebbly mudstone. The lonestones contained in the Singa Formation have been interpreted to be of glacial origin [10,18-20]. The presence of matrix-hosted fresh feldspars, granitic clasts (up to $10 \mathrm{~cm}$ in diameter), angular limestone clasts, and brachiopods indicates that the Singa Formation was deposited in a glacial-marine environment [21].

The authors of [20] documented the presence of radiolarian in black siliceous mudstone and cherts, of Ordovician-Silurian age, Upper Devonian-Lower Carboniferous, and Middle Permian age for the Singa Formation. The Singa Formation detrital zircon age was calculated by the method of U-Pb zircon dating and gave an age of $418 \mathrm{Ma}$ [8]. All other agerelated evidence such as fossils and glacial deposit exposures point to a Pennsylvanian to early Permian age. The fossil age is also supported by data from the Kaeng Krachan Group (initially Phuket Group in Thailand) discovered off the border Malaysia-Thailand [19,20].

The presence of megaclast-bearing units in the Upper Paleozoic of Southeast Asia likely indicates glacial marine deposits [18]. Regionally, these glacial deposits extend from 
Sumatra to Myanmar (Burma) over $2000 \mathrm{~km}$. The deposits are related to the activities of wide continental ice sheet. Gondwana is known to have been a land where glacial episodes occurred along the Sibumasu terrane.

The Singa Formation which contains pebbly dark-colored mudstones can be correlated to other glacial deposits cropping out in the Kaeng Krachan Formation (Phuket Group) of Thailand, the Bohorok Formation of Sumatra and Mergui, Martaban (Indonesia), and Lebyin Groups of Burma (Myanmar) [21]. This formation is interpreted as having been deposited in a shallow marine environment under the influence of polar glaciation and glacial melting [12]. At a regional scale, the Singa Formation is correlated to the Kubang Pasu Formation, exposures of which are found in the northwestern part of Peninsular Malaysia [12]. The lithological log and the outcrop and hand specimen features are shown in Figures 2 and 3. Brachiopod fossils were found in the Singa Formation suggesting cold-water conditions of the Gondwanaland seas.
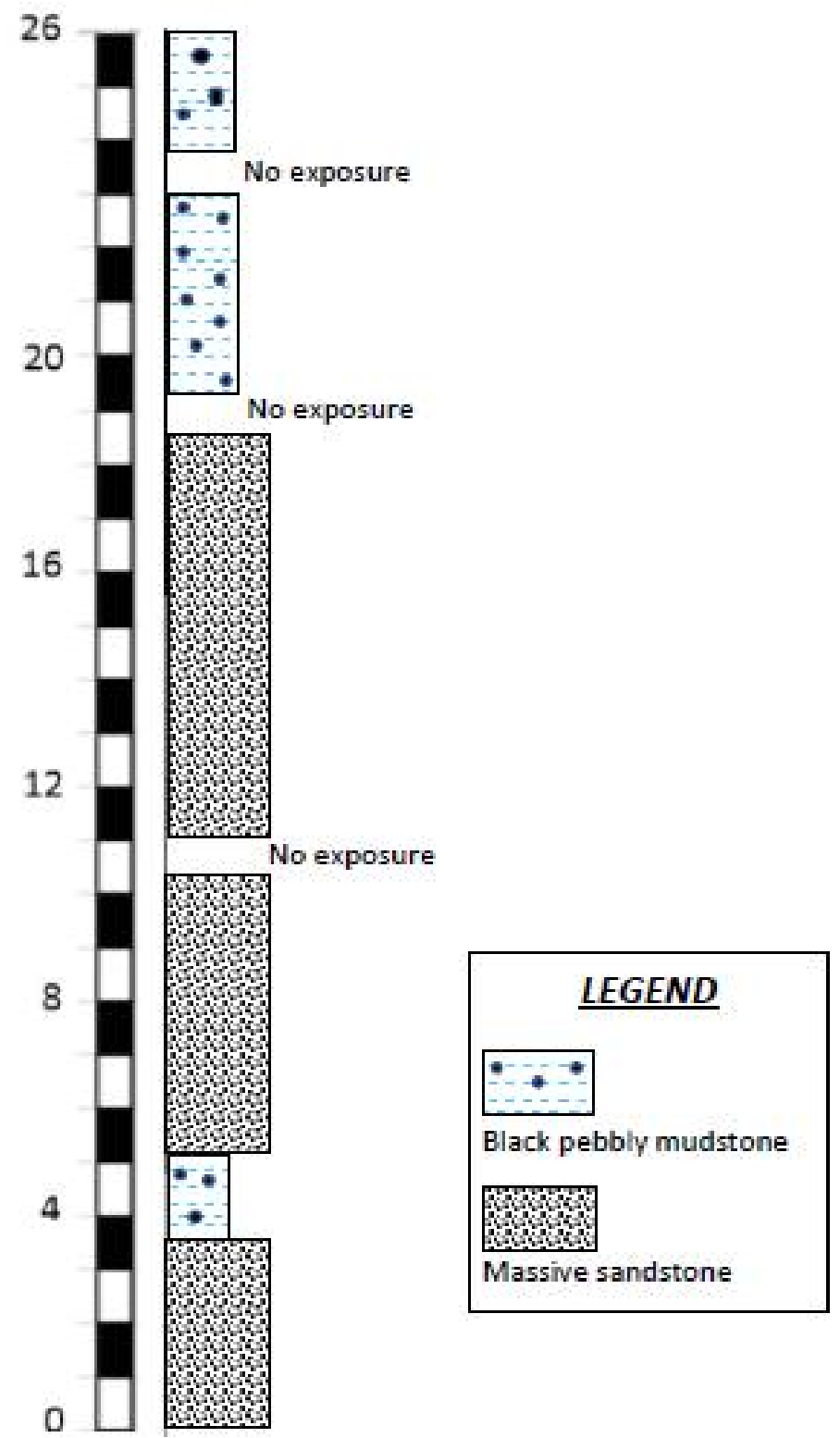

Figure 2. Lithologic log for the Singa Formation section in Langkawi Islands, Malaysia. 

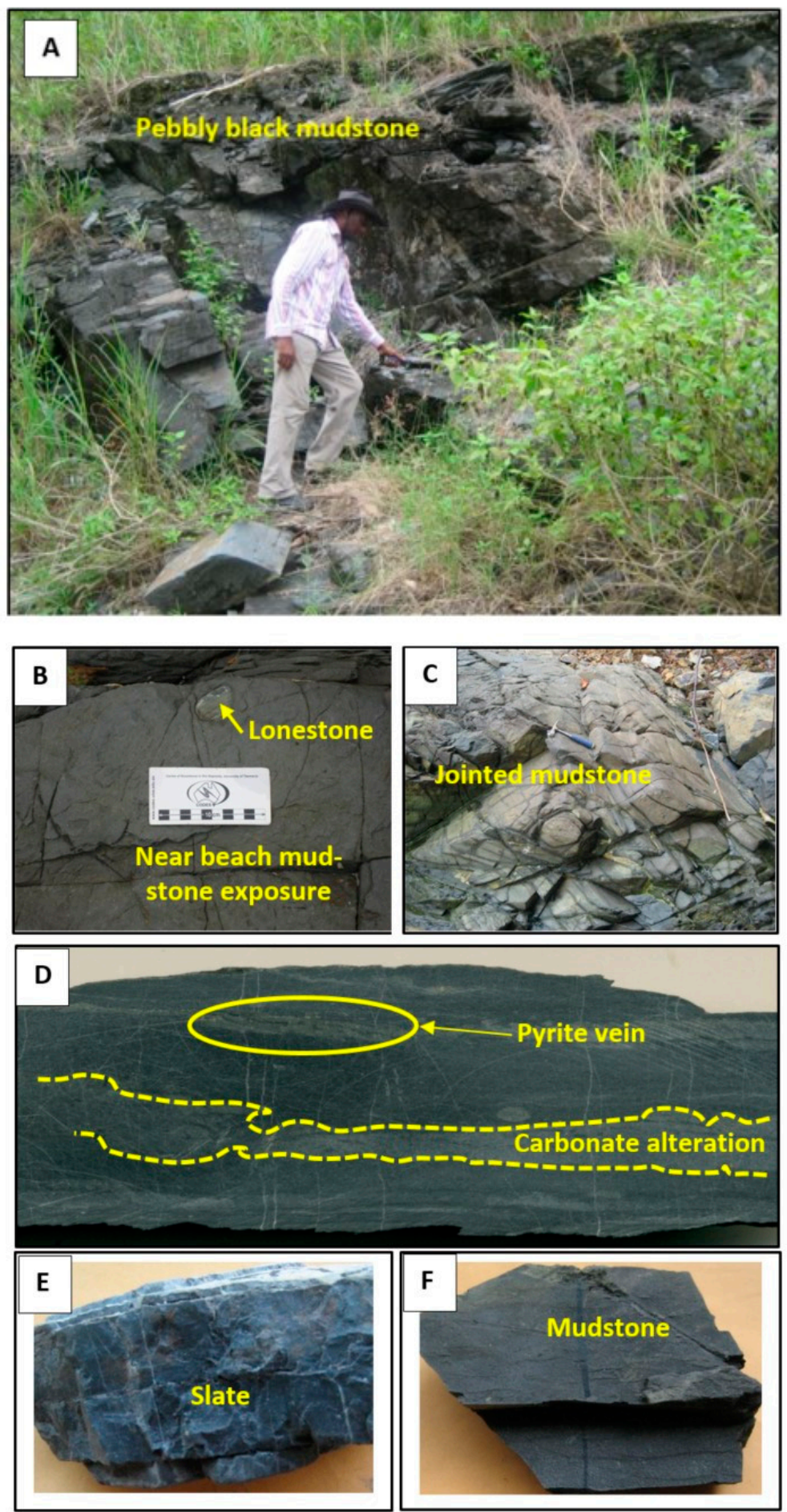

Figure 3. Photographs of outcrops and hand specimen of Singa Formation from Langkawi Islands, Malaysia. (A) Black mudstones exposure. (B) Presence of lonestones on a bedding plan of the mudstones. (C) Highly jointed mudstone. (D) Pyrite veins, carbonate alteration, and thin calcite veins cross-cutting bedding. (E) Slaty mudstone. (F) Black mudstone.

\section{Methods of Study}

The methods described here include field work and the laboratory work carried out at the Centre for Ore Deposit and Earth Science (CODES), University of Tasmania, Australia. Lithologic logging and sampling were undertaken. To record mapping data, the global positioning system device (GPS) was utilized to keep a full record of latitude 
and longitude from each sampling site. In this study, coordinate systems are in UTM (Universal Transverse Mercator), zone 47, northern hemisphere (using Map datum-WGS 84), latitude-longitude (using Map datum-Kertau 1948) system.

\subsection{X-ray Fluorescence Analysis (XRF)}

A total of 13 black mudstone samples were analyzed using $X$-ray fluorescence analytical technique (XRF), total organic carbon (TOC) method, and laser ablation inductively coupled plasma (LA ICP-MS) on pyrite at the Centre for Ore Deposit and Earth Sciences (CODES) Analytical Laboratories, University of Tasmania. The samples were collected from fresh and slightly weathered outcrop. The instrument used for this analysis is Panalytical Axios Advanced X-ray Spectrometer. It is equipped with Rh X-ray Tube operating at $4 \mathrm{~kW}$ (max), and PX-10, LiF 220, PX-1, curved PE002, and curved Ge111 analyzers. The types of detectors are Gas flow proportional counters with P10 gas (10\% methane argon), a sealed Xe Duplex and Scintillation Counter.

After assessment of Loss on Ignition (LOI, wt.\%), fused disks were prepared for analysis of a set of major elements ( $\mathrm{Na}, \mathrm{Mg}, \mathrm{Al}, \mathrm{Si}, \mathrm{P}, \mathrm{S}, \mathrm{K}, \mathrm{Ca}, \mathrm{Ti}, \mathrm{Mn}, \mathrm{Fe})$.

The $32 \mathrm{~mm}$ fusion discs were prepared at 1100 degrees $\mathrm{C}$ in $5 \% \mathrm{Au} / 95 \% \mathrm{Pt} \mathrm{cru}-$ cibles /molds using a mixture of $0.500 \mathrm{~g}$ of sample $4.500 \mathrm{~g}$ of 12-22 Flux (Lithium TetraborateMetaborate mix), and $0.0606 \mathrm{~g} \mathrm{LiNO}_{3}$ for silicates. Sulfide bearing samples had a different mix with the component $\mathrm{LiNO}_{3}$ as oxidizing agent and the mix is pre-ignited at the temperature of 700 degrees $C$ for a duration of $10 \mathrm{~min}$.

Preparation of pressed powder pills weighing $10 \mathrm{~g}$ each was done using PVP-MC Binder to determine trace element composition. Corrections for mass absorption were calculated using Panalytical Super-Q software with its classic calibration model and alpha coefficients. In house inter-element corrections were also applied. Calibration was done on pure element oxide mixed in pure silica, along with International and Tasmanian reference rocks. The estimated detection limits of XRF analysis vary between 0.5 and $4 \mathrm{ppm}$, except sulfur: Sc (1.5 ppm), Ba (4 ppm), V (3 ppm), Cr (1 ppm), Ni (1 ppm), Cu (1 ppm), Zn (1 ppm), Ga (2 ppm), As (3 ppm), Se (1 ppm), Rb (0.5 ppm), Sr ( 1 ppm), Y (1 ppm), $\mathrm{Zr}$ (1 ppm), Nb (0.5 ppm), Mo (0.5 ppm), Ag (2 ppm), Sn (1 ppm), Sb (2 ppm), Te (2 ppm), $\mathrm{Tl}$ (2 ppm), Pb (1 ppm), Bi (2 ppm), U (2 ppm), and Th (2 ppm). The detection limit for total sulfur content is $0.01 \mathrm{wt} . \%$. In tables, dashes represent undetected trace elements or below detection limit (bdl). Previous research works documented that gold contents ranged from 2.3 to $57 \mathrm{ppb} \mathrm{Au}$ indicative of crustal levels of gold in most shales [22-24]. This gold range from previous studies is compared to the gold range in this study to verify if there are some anomalous values in the Singa black mudstones.

\subsection{Total Organic Carbon (TOC) and Isotope Determination}

The TOC calculation was performed as follows: samples were crushed and milled using a tungsten-carbide mill. Approximately $10 \mathrm{~g}$ of the samples was then measured using a crucible. Afterwards, the samples and crucible were left in an oven during the night to dry in a heat up to $450{ }^{\circ} \mathrm{C}$. The combustion of the sample served to get rid of the organic carbon component and preserve the inorganic carbon component as tested by Krom and Berner [25]. The unashed half was then processed separately by measuring the total carbon which was a combination of the organic and inorganic carbon (calcium carbonate) contents in each powder sample. Finally, the total organic carbon was calculated as the difference between total carbon and inorganic carbon [25].

The analysis of $\delta^{13} \mathrm{C}$ and $\delta^{34} \mathrm{~S}$ was done by NCS combustion. It is a capability of the vario MICRO, ISOTOPE, and PYRO cubes. The vario PYRO cube has the following setup for NCS mode: two packed reactor tubes (combustion and reduction), two 'purge and trap' desorption columns (for $\mathrm{SO}_{2}$ and $\mathrm{CO}_{2}$ ) and an inlet for both the sample and reference gas to enter the IsoPrime100 IRMS. After combustion, the bulk sample gas goes through the system and columns and is stripped of $\mathrm{H}_{2} \mathrm{O}$, in the water traps, as well as $\mathrm{SO}_{2}$ and $\mathrm{CO}_{2}$, in the 'purge and trap' columns. The $\mathrm{N}_{2}$ component gas was not trapped in a column and 
was the first to enter the IRMS. After the $\mathrm{N}_{2}$ reference and sample peaks were collected the $\mathrm{CO}_{2}$ desorption column was heated to $110^{\circ} \mathrm{C}$. The $\mathrm{CO}_{2}$ sample gas was released, passing through a second water trap and into the IRMS. The final gas to be released was $\mathrm{SO}_{2}$ which occurred when the desorption column was heated to $220^{\circ} \mathrm{C}$; this sample gas then bypassed the $\mathrm{CO}_{2}$ column (where it may be retained), transited a second water trap, and passed through the IRMS. The dilutor can be used to lower the gas loads entering the IRMS source. This 'purge and trap' technique of gas release on the vario PYRO is superior to standard GC separation techniques on other EA systems. This method allows user control over timing, without peak broadening due to slow-release times between different gas species.

\subsection{Laser Ablation Inductively Coupled Mass Spectrometry (LA ICP-MS)}

The trace element contents in the selected pyrite samples were analyzed by laser ablation-inductively coupled-mass spectrometry (LA-ICP-MS) at CODES, University of Tasmania. The instrument combines a Resolution $193 \mathrm{~nm}$ excimer laser, mixed with an Agilent 7700x ICP-MS. To map trace element distribution, signal was acquired in timeresolved mode with a laser beam of $5 \mu \mathrm{m}$, laser fluence of $\sim 3.5 \mathrm{~J} / \mathrm{cm}^{2}$, and a repetition rate of $5 \mathrm{~Hz}$. The signal from the carrier gas with no ablation was regularly acquired to correct for instrumental background.

The following reference materials were used for primary calibration and assessment of elemental contents: STDGL2b2 [26] and STDGL3 $[27,28]$ for siderophile and chalcophile elements, GSD-1G [29] for lithophile elements, and PPP-1 pyrite for sulfur [30]. The spot sizes used were 10,15,20, and $22 \mu \mathrm{m}$. Line analyses were done with laser spot sizes of 15 and $25 \mu \mathrm{m}$ and a firing rate of $5 \mathrm{~Hz}$, moving at a speed of $3 \mu$ /s with laser energy of $3.5 \mathrm{~J} / \mathrm{cm}^{2}$. The spot size of $22 \mu \mathrm{m}$ was used and each line was initially ablated without analyzing. The backgrounds were recorded before each image and subtracted from each analysis line [31]. Twenty-nine elements were analyzed on pyrite grains including: $\mathrm{Na}, \mathrm{Mg}$, $\mathrm{Al}, \mathrm{Si}, \mathrm{K}, \mathrm{Ca}, \mathrm{Ti}, \mathrm{V}, \mathrm{Cr}, \mathrm{Mn}, \mathrm{Fe}, \mathrm{Co}, \mathrm{Ni}, \mathrm{Cu}, \mathrm{Zn}, \mathrm{As}, \mathrm{Se}, \mathrm{Mo}, \mathrm{Ag}, \mathrm{Sb}, \mathrm{Te}, \mathrm{W}, \mathrm{Pt}, \mathrm{Au}, \mathrm{Hg}, \mathrm{Tl}, \mathrm{Pb}$, $\mathrm{Bi}$, and $\mathrm{U}$.

\subsection{Sulfur Isotope Analysis}

Mudstone samples were analyzed by the conventional technique at the Central Science Laboratory Facility (CSL), University of Tasmania, Australia. For the conventional technique, pyrite grains were drilled out using a dentist's drill to produce powdered samples weighing about $10-25 \mathrm{mg}$. The powdered samples were submitted to the Central Science Laboratory for analysis. The isotopic compositions for S34/S32 were determined by combustion with cuprous oxide method [32]. The Canon Diablo Troilite (CDT) international standard was used to monitor the raw sulfur isotopic data. An accuracy of $\pm 0.15 \%$ was obtained by the conventional method at the Central Science Laboratory, University of Tasmania, Australia.

\section{Textures of Pyrite and Associated Minerals}

Petrographic investigation has shown six pyrite types for the Singa Formation: (1) early generations of pyrite which include disseminated framboidal pyrites (pyrite 1) $(<20 \mu \mathrm{m})$, up to $30 \mu \mathrm{m}$ wide and $50 \mu \mathrm{m}$ long diagenetic aggregates of pyrite nodules (pyrite 2), clusters of euhedral clean pyrite (pyrite 3); (2) late generations of pyrite that are comprised of coarse-grained, subhedral pyrite (pyrite 4), inclusion-rich pyrite (pyrite 5), and coarse euhedral clean pyrite (pyrite 6) (Figure 4). Pyrrhotite is also present and associated with pyrite 6. Chalcopyrite is found intergrown with pyrite 4 and pyrite 5 . 

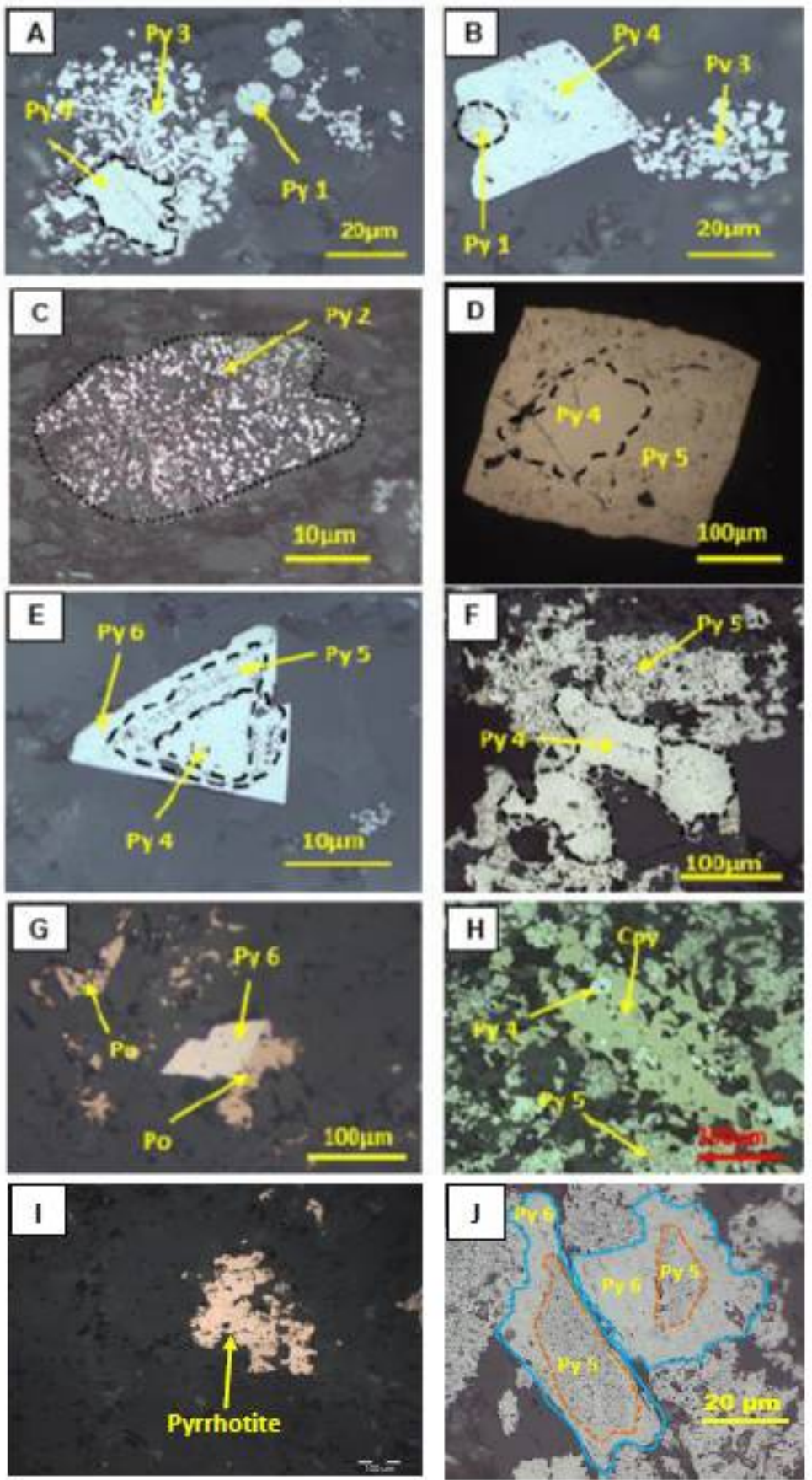

Figure 4. Textural characteristics of pyrite with other sulfide minerals in the Singa Formation, Malaysia. (A) Diagenetic pyrites (py 1), microcrystals of euhedral pyrite 3, and clean subhedral pyrite 4 (sample LA-3812A). (B) Diagenetic pyrite 1 overgrown by euhedral pyrite 4 and associated with pyrite 3 (sample LA-3812B). (C) Aggregate of micro-nodules of pyrite (py 2) (sample LA-3812C). (D) Subhedral pyrite 4 overgrown by pyrite 5 (sample LA-5312). (E) Zoned pyrite grain showing pyrite 4 (core) overgrown by pyrite 5, which in turn, rimmed by pyrite 6 (sample LA-3812A). (F) Pyrite 4 associated with pyrite 5 (sample LA 4112). (G) Pyrite 6 and pyrrhotite (po) in the form of patches which size ranges from 5 to $200 \mu \mathrm{m}$ across (sample LA-4112). (H) Chalcopyrite (cpy) formed around microcrystals of pyrite 4 associated with pyrite 5 (sample LA-4112). Sample identification: LA-3812 and LA-4112 represent black shales; LA-5312 is a sandstone sample. (I) Pyrrhotite in slate (sample LA-4212). (J) Inclusion-rich pyrite 5 overgrown by pyrite 6 (sample LA-4212). 


\section{Results}

\subsection{Major and Trace Element Composition}

The geochemical analyses of the mudstones (samples number: SBS1-SBS10) are presented in Tables 1 and 2. Results of mean values in the black mudstones are $\mathrm{SiO}_{2}(65.02$ wt.\%), $\mathrm{Al}_{2} \mathrm{O}_{3}$ (14.48 wt.\%), $\mathrm{MgO}$ (2.62 wt.\%), $\mathrm{Na}_{2} \mathrm{O}$ (1.91 wt.\%), $\mathrm{K}_{2} \mathrm{O}$ (83.56 wt.\%), $\mathrm{Fe}_{2} \mathrm{O}_{3}$ (5.75 wt.\%), $\mathrm{MnO}$ (0.08 wt.\%), $\mathrm{CaO}$ (2.02 wt.\%), $\mathrm{TiO}_{2}$ (0.77 wt.\%), and $\mathrm{P}_{2} \mathrm{O}_{5}$ (0.16 wt.\%) (Table 3). A spider plot of major elements in the mudstones compared with Post Archean Australian shales (PAAS) is presented in Figure 5 to show the relative abundance of the major elements. The black mudstones also show minor enrichment in $\mathrm{Na}_{2} \mathrm{O}$ with two samples that are enriched and depleted in $\mathrm{CaO}$ (Figure 6). Additionally, total organic carbon (TOC) content of black shales ranges from 0.02 to $0.37 \mathrm{wt} . \%$ (mean $0.21 \mathrm{wt} . \%$ ). Although trace element composition shows a depletion in $\mathrm{Cu}, \mathrm{Sr}$, and Mo contents, the mudstones have a trace element pattern almost identical to PAAS (Figure 6).

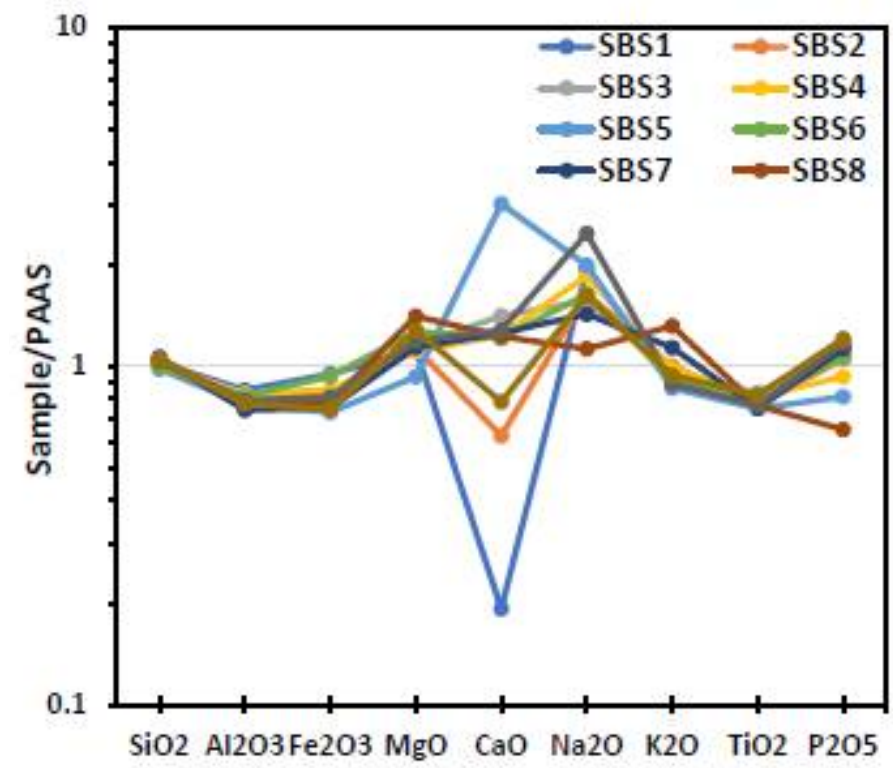

Figure 5. Major elements in black mudstones normalized to PAAS.

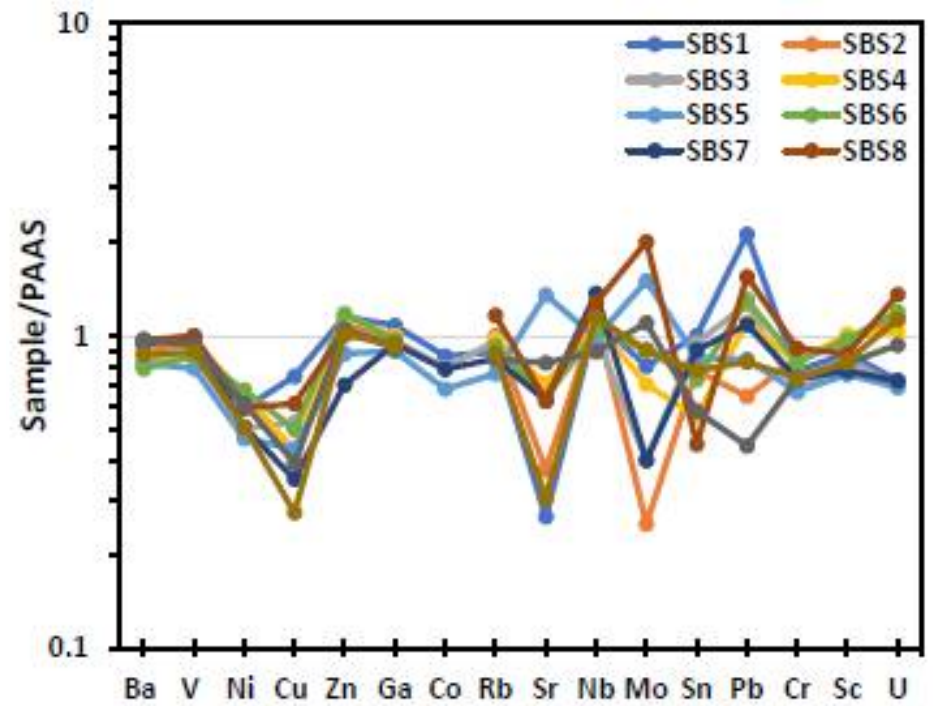

Figure 6. Trace elements in black mudstones normalized to PAAS. 
Table 1. Trace element, aluminum, and total organic carbon contents in black mudstones from the Singa Formation, Langkawi Malaysia. This table comprises ten samples (SM1-10) that were analyzed. Unit: Al (wt.\%); TOC (wt.\%); S (wt.\%); $\mathrm{Au}(\mathrm{ppb})$; other trace elements (ppm); $\mathrm{BDL}=$ below detection limit.

\begin{tabular}{|c|c|c|c|c|c|c|c|c|c|c|}
\hline Element & SMS1 & SMS2 & SMS3 & SMS4 & SMS5 & SMS6 & SMS7 & SMS8 & SMS9 & SMS10 \\
\hline $\mathrm{Al}$ & 8.50 & 8.19 & 8.02 & 8.29 & 7.71 & 8.16 & 7.41 & 7.84 & 7.92 & 7.76 \\
\hline $\mathrm{Au}$ & 46.30 & 47.58 & 61.65 & 53.25 & 45.76 & 47.76 & 65.28 & 57.08 & 41.70 & 39.63 \\
\hline TOC & 0.20 & 0.23 & 0.37 & 0.24 & 0.25 & 0.35 & 0.10 & 0.11 & 0.02 & 0.13 \\
\hline S & 0.03 & 0.005 & 0.01 & 0.09 & 0.08 & 0.06 & 0.005 & 0.39 & 0.005 & 0.005 \\
\hline $\mathrm{Ba}$ & 604.00 & 581.00 & 542.00 & 573.00 & 530.00 & 511.00 & 621.00 & 631.00 & 632.00 & 566.00 \\
\hline $\mathrm{V}$ & 143.00 & 142.00 & 129.00 & 129.00 & 118.00 & 129.00 & 150.00 & 149.00 & 140.00 & 133.00 \\
\hline $\mathrm{Ni}$ & 32.18 & 35.70 & 27.92 & 34.90 & 25.79 & 36.90 & 28.07 & 32.40 & 33.40 & 28.10 \\
\hline $\mathrm{Cu}$ & 37.09 & 21.30 & 26.40 & 21.50 & 21.76 & 25.10 & 17.39 & 30.30 & 19.60 & 13.60 \\
\hline $\mathrm{Zn}$ & 97.67 & 91.90 & 85.34 & 97.90 & 74.57 & 100.00 & 59.19 & 88.20 & 89.20 & 85.90 \\
\hline $\mathrm{Ga}$ & 21.57 & 19.80 & 19.35 & 19.90 & 17.88 & 19.40 & 18.82 & 18.80 & 18.20 & 18.70 \\
\hline As & 54.70 & 26.20 & 13.00 & 12.50 & 6.70 & 12.00 & 10.00 & 17.20 & 11.80 & 15.60 \\
\hline $\mathrm{Co}$ & 19.80 & BDL & 18.14 & BDL & 15.55 & BDL & 17.96 & $\mathrm{BDL}$ & BDL & BDL \\
\hline $\mathrm{Rb}$ & 142.14 & 160.00 & 153.67 & 156.00 & 120.90 & 149.00 & 135.15 & 186.00 & 135.00 & 141.00 \\
\hline $\mathrm{Sr}$ & 52.70 & 74.40 & 131.68 & 141.00 & 270.25 & 127.00 & 124.19 & 124.00 & 164.00 & 60.50 \\
\hline $\mathrm{Y}$ & 33.25 & 34.30 & 31.40 & 30.80 & 30.30 & 32.20 & 30.98 & 28.10 & 29.90 & 32.50 \\
\hline $\mathrm{Zr}$ & 225.00 & 251.00 & 250.20 & 234.00 & 241.30 & 228.00 & 282.30 & 229.00 & 228.00 & 278.00 \\
\hline $\mathrm{Nb}$ & 21.40 & 21.90 & 19.10 & 20.60 & 18.70 & 19.90 & 26.00 & 24.20 & 16.90 & 21.80 \\
\hline Mo & 0.80 & 0.25 & 0.40 & 0.70 & 1.50 & 0.90 & 0.40 & 2.00 & 1.10 & 0.90 \\
\hline $\mathrm{Ag}$ & 0.15 & 1.00 & 0.15 & 1.00 & 0.15 & 1.00 & 0.15 & 1.00 & 1.00 & 1.00 \\
\hline Sn & 4.00 & 3.10 & 3.80 & 2.20 & 3.40 & 2.90 & 3.60 & 1.80 & 2.30 & 3.10 \\
\hline $\mathrm{Sb}$ & 6.70 & 4.20 & 0.60 & 1.00 & 0.60 & 1.00 & 0.40 & 1.00 & 1.00 & 1.00 \\
\hline $\mathrm{Te}$ & 0.10 & 1.00 & 0.05 & 1.00 & 0.05 & 1.00 & 0.10 & 1.00 & 1.00 & 1.00 \\
\hline Th & 19.36 & 21.40 & 17.01 & 18.60 & 15.89 & 19.50 & 19.87 & 20.20 & 14.10 & 15.70 \\
\hline $\mathrm{Pb}$ & 42.30 & 12.80 & 25.25 & 22.10 & 16.81 & 26.10 & 21.73 & 30.90 & 8.90 & 16.50 \\
\hline $\mathrm{Bi}$ & 0.60 & 1.00 & 0.30 & 1.00 & 0.30 & 1.00 & 0.20 & 1.00 & 1.00 & 1.00 \\
\hline $\mathrm{Cr}$ & 84.70 & 93.60 & 72.70 & 90.40 & 72.90 & 90.60 & 80.10 & 100.40 & 80.00 & 80.50 \\
\hline Th & 19.36 & 21.40 & 17.01 & 18.60 & 15.89 & 19.50 & 19.87 & 20.20 & 14.10 & 15.70 \\
\hline $\mathrm{Sc}$ & 14.06 & 15.20 & 13.43 & 16.20 & 12.05 & 15.60 & 12.35 & 14.00 & 13.10 & 13.30 \\
\hline $\mathrm{La}$ & 46.67 & 36.60 & 43.75 & 46.30 & 45.23 & 44.90 & 49.34 & 46.80 & 33.90 & 39.90 \\
\hline $\mathrm{U}$ & 2.23 & 3.50 & 2.15 & 3.20 & 2.12 & 3.70 & 2.22 & 4.20 & 2.90 & 3.50 \\
\hline $\mathrm{Nd}$ & 41.07 & 31.40 & 37.70 & 41.20 & 39.41 & 37.30 & 40.16 & 35.70 & 31.30 & 35.30 \\
\hline
\end{tabular}

Table 2. Major element composition of black mudstones from the Singa Formation.

\begin{tabular}{|c|c|c|c|c|c|c|c|c|c|c|c|c|}
\hline Lithology & Sample & $\mathrm{SiO}_{2}$ & $\mathrm{Al}_{2} \mathrm{O}_{3}$ & $\mathrm{Fe}_{2} \mathrm{O}_{3}$ & $\mathrm{MgO}$ & $\mathrm{CaO}$ & $\mathrm{Na}_{2} \mathrm{O}$ & $\mathrm{K}_{2} \mathrm{O}$ & $\mathrm{TiO}_{2}$ & $\mathrm{P}_{2} \mathrm{O}_{5}$ & Loss & Total \\
\hline Mudstone & SMS1 & 64.16 & 16.07 & 6.85 & 2.46 & 0.25 & 2.32 & 3.29 & 0.83 & 0.18 & 3.59 & 100 \\
\hline Mudstone & SMS2 & 64.53 & 15.49 & 6.05 & 2.49 & 0.81 & 1.96 & 3.7 & 0.771 & 0.168 & 3.7 & 99.669 \\
\hline Mudstone & SMS3 & 63.86 & 15.17 & 5.79 & 2.52 & 1.83 & 1.87 & 3.54 & 0.83 & 0.17 & 4.32 & 99.9 \\
\hline Mudstone & SMS4 & 62.58 & 15.67 & 6.15 & 2.43 & 1.6 & 2.22 & 3.63 & 0.811 & 0.149 & 4.25 & 99.49 \\
\hline Mudstone & SMS5 & 61.57 & 14.58 & 5.28 & 2.05 & 3.92 & 2.38 & 3.19 & 0.75 & 0.13 & 5.75 & 99.6 \\
\hline Mudstone & SMS6 & 62.55 & 15.43 & 6.74 & 2.79 & 1.57 & 1.96 & 3.38 & 0.787 & 0.17 & 4.32 & 99.697 \\
\hline Mudstone & SMS7 & 66.72 & 14.01 & 5.44 & 2.5 & 1.63 & 1.71 & 4.2 & 0.75 & 0.18 & 2.57 & 99.71 \\
\hline Mudstone & SMS8 & 64.77 & 14.82 & 5.64 & 3.09 & 1.59 & 1.35 & 4.86 & 0.764 & 0.104 & 2.42 & 99.408 \\
\hline Mudstone & SMS9 & 65.59 & 14.97 & 5.87 & 2.61 & 1.66 & 2.96 & 3.28 & 0.765 & 0.187 & 1.48 & 99.372 \\
\hline Mudstone & SMS10 & 65.81 & 14.66 & 5.38 & 2.81 & 1.02 & 1.94 & 3.48 & 0.821 & 0.193 & 1.86 & 97.974 \\
\hline Shale & PAAS & 62.8 & 18.9 & 7.22 & 2.2 & 1.3 & 1.2 & 3.7 & 1 & 0.16 & & \\
\hline
\end{tabular}

\subsection{Pyrite Composition, Organic Carbon, and Sulfur Isotopes}

Trace element composition of pyrite contained in the mudstones is presented in Table 3. Singa pyrite framboids and micro-euhedral pyrites (pyrites 1,2, and 3) have gold content in the range of 0.05-1.6 ppm. However, euhedral pyrites (pyrites 4, 5, and 6) indicate low gold contents ranging up to $0.5 \mathrm{ppm}$. Early pyrite generations (pyrites 1, 2, and 3) have elevated As, Te, Mo, Sb, Co, and Se relative to late pyrite generations (pyrites 4, 5, and 6). Elements such as $\mathrm{Cr}$ and $\mathrm{Sn}$ were not detected in the pyrite generations. Singa euhedral pyrite 6 is markedly depleted in Mo compared to earlier generations (pyrites 1, 2, and 
3). Compared to worldwide diagenetic (WDP) pyrites [33], Singa early pyrites (pyrites 1 , 2, and 3) are enriched in $\mathrm{Au}, \mathrm{Co}, \mathrm{Zn}$, and Te and depleted in Ag (Figure 7). Mean trace element variation composition in pyrite from the Singa Formation, Malaysia is shown in Figure 8. Organic carbon isotope signature for the pyritic mudstones ranges from $-23.9 \%$ to $-5.8 \%$. Additionally, the $\delta^{34} \mathrm{~S}$ values for pyrite grains vary from $-24.6 \%$ to $+6.2 \%$ o (Table 4).

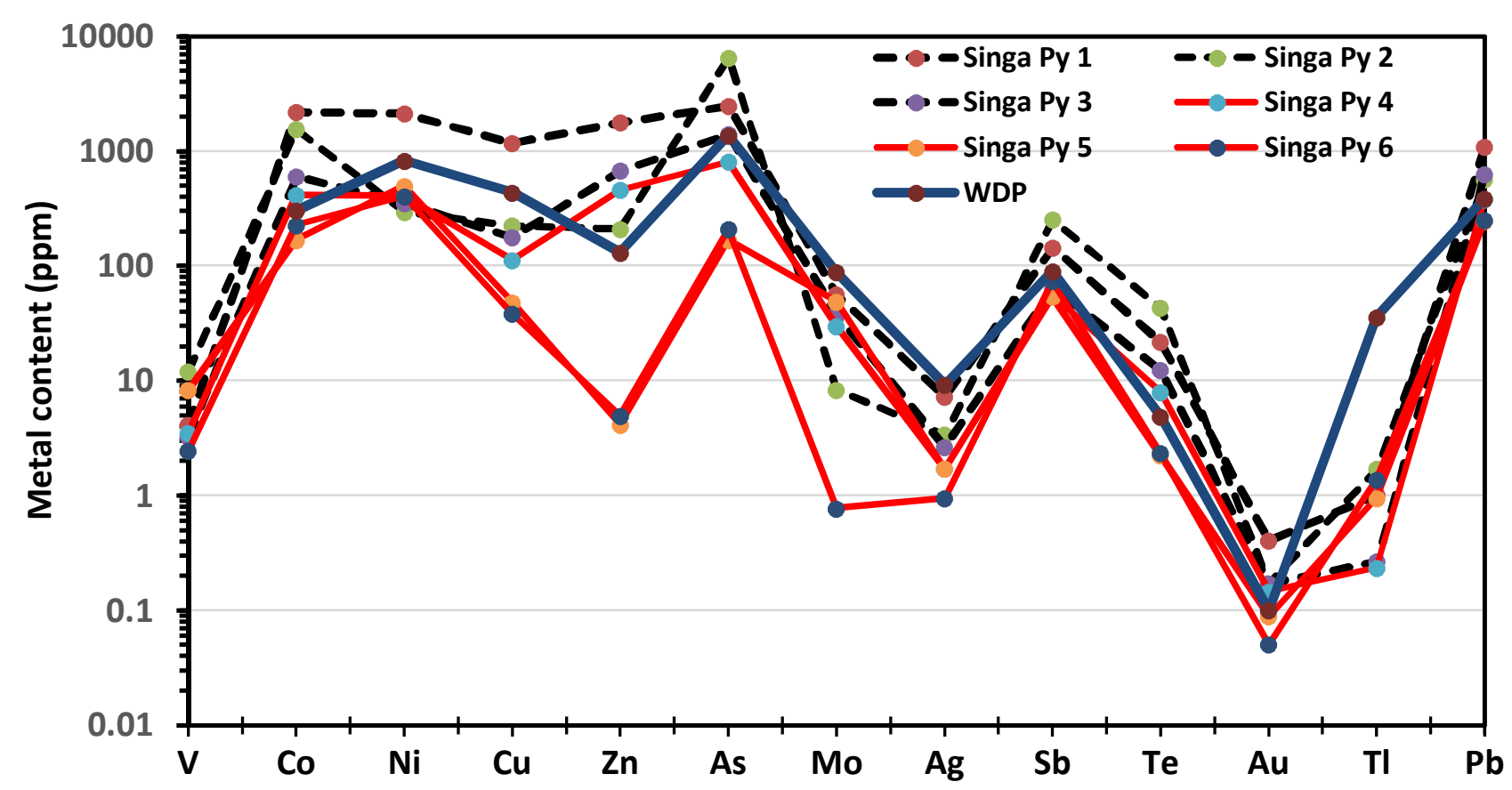

Figure 7. Mean trace element composition in pyrite (this study) from the Singa Formation, Malaysia. Note: WDP = World diagenetic pyrite (Gregory et al., 2015) [33].

Table 3. Pyrite trace element chemistry from the mudstones of the Singa Formation, Langkawi, Malaysia. BDL = below detection limit.

\begin{tabular}{|c|c|c|c|c|c|c|c|c|c|c|c|c|c|c|c|c|}
\hline Pyrite ID & $\mathbf{V}$ & Co & $\mathrm{Ni}$ & $\mathrm{Cu}$ & $\mathrm{Zn}$ & As & Se & Mo & Ag & $\mathrm{Sb}$ & $\mathrm{Te}$ & $\mathrm{Au}$ & $\mathrm{Tl}$ & $\mathbf{P b}$ & $\mathrm{Cd}$ & $\mathbf{B i}$ \\
\hline Py 1_A & 4.1 & 3767 & 956 & 775 & 107 & 3643 & 67 & 76.38 & 12 & 221 & 38 & 0.9 & 2.8 & 977 & 1.7 & 234 \\
\hline Py 1_B & 3.9 & 2714 & 903 & 293 & 146 & 3552 & BDL & 66.52 & 11 & 184 & 30 & 0.72 & 1.9 & 664 & 1.6 & 178 \\
\hline Py 1_C & 2 & 2296 & 1720 & 8325 & 1331 & 2330 & BDL & 48.07 & 11 & 131 & 25 & 0.17 & 2.15 & 1635 & 2.8 & 119 \\
\hline Py 1 1_D & 21.4 & 4193 & 765 & 315 & 125 & 3516 & BDL & 105.01 & 20 & 214 & 36 & 1.61 & 0.522 & 992 & 0.5 & 237 \\
\hline Py 1_E & 1.6 & 1095 & 663 & 145 & 182 & 1063 & 68 & 43.56 & 3 & 53 & 15 & 0.07 & 0.34 & 604 & 2 & 57 \\
\hline Py 1_F & 13.6 & 5344 & 720 & 4341 & 687 & 5033 & BDL & 41.69 & 14 & 215 & 37 & 0.59 & 2.24 & 1738 & 4 & 211 \\
\hline Py 1 _G & 3.5 & 2116 & 17,721 & 311 & 1432 & 1461 & 99 & 34.4 & 10 & 218 & 23 & 0.24 & 1.62 & 8903 & 4 & 100 \\
\hline Py 1_H & 1.6 & 1788 & 13,812 & 244 & 1461 & 2138 & 72 & 26.8 & 4 & 197 & 15 & 0.1 & 1.15 & 6309 & 22 & 91 \\
\hline Py 1_I & 1.1 & 650 & 13,551 & 334 & 538 & 993 & 60 & 40.95 & 5 & 266 & 8 & 0.24 & 1.28 & 2269 & 1.68 & 46 \\
\hline Py 1_J & 3.3 & 2860 & 413 & 428 & 2282 & 3351 & BDL & 78.01 & 8 & 247 & 43 & 0.33 & 1.94 & 908 & 19 & 226 \\
\hline Py $1 \_K$ & 0 & 787 & 7046 & 280 & 460 & 1747 & 50 & 23.1 & 3 & 295 & 22 & 0.26 & 1.44 & 2166 & 2 & 104 \\
\hline Py 1_L & 2.4 & 1121 & 323 & 206 & 475 & 4226 & BDL & 28.66 & 5 & 89 & 7 & 0.31 & 0.57 & 600 & 1.84 & 58 \\
\hline Py 1_M & 1.9 & 4764 & 910 & 691 & 1990 & 2605 & BDL & 40.23 & 4 & 146 & 17 & 0.34 & 0.62 & 1149 & 7 & 108 \\
\hline Py $1 \_N$ & 1.2 & 413 & 115 & 90 & 329 & 4790 & BDL & 28.69 & 4 & 51 & 3.85 & 0.14 & 0.211 & 242 & 2.17 & 35 \\
\hline Py 1_O & 0.7 & 3182 & 1022 & 371 & 342 & 3427 & BDL & 95.05 & 6 & 245 & 26 & 0.58 & 1.29 & 784 & 1.84 & 84 \\
\hline Py 1_P & 0 & 296 & 97 & 85 & 198 & 454 & 66 & 216.78 & 3 & 46 & 3.38 & 0.13 & 0.185 & 1515 & 1.91 & 33 \\
\hline Py 1_Q & 0 & 380 & 150 & 68 & 79 & 413 & 42 & 33.98 & 1 & 42 & 7 & 0.06 & 0.17 & 937 & 0.77 & 28 \\
\hline Py 1_R & 1.2 & 885 & 480 & 150 & 343 & 800 & 72 & 12.22 & 3 & 63 & 9 & 0.13 & 0.18 & 748 & 1.88 & 50 \\
\hline Py 1_S & 3.4 & 137 & 148 & 46 & 167 & 470 & BDL & 5.53 & 0.5 & 34 & 3.52 & 0.13 & 0.19 & 722 & 1.98 & 12 \\
\hline Py 1_T & 0.1 & 128 & 46 & 66 & 461 & 298 & BDL & 9.78 & 0.5 & 32 & 3.61 & 0.14 & 0.2 & 785 & 2.04 & 7 \\
\hline Py 1_U & 0.9 & 791 & 234 & 218 & 1049 & 832 & BDL & 41.47 & 4 & 86 & 3.23 & 0.12 & 0.18 & 795 & 1.82 & 31 \\
\hline Py 1_V & 0 & 232 & 116 & 110 & 474 & 435 & BDL & 19.75 & 2 & 65 & 3.35 & 0.13 & 0.18 & 591 & 1.89 & 19 \\
\hline Py 1_W & 4.2 & 269 & 371 & 130 & 784 & 682 & 65 & 27.05 & 1 & 42 & 6 & 0.05 & 0.07 & 779 & 1 & 35 \\
\hline Py 1_x & 1.1 & 3654 & 939 & 307 & 114 & 3716 & 14 & 113.19 & 13 & 237 & 44 & 0.69 & 2.79 & 0.4 & 0 & 213 \\
\hline Py 1_Y & 5.8 & 2875 & 664 & 12,574 & 1592 & 2800 & 42 & 56.56 & 12 & 216 & 38 & 0.65 & 1.8 & 3.3 & 3 & 166 \\
\hline Py $1 \_Z$ & 10.4 & 3572 & 898 & 299 & 352 & 3834 & BDL & 126.63 & 12 & 240 & 41 & 0.83 & 1.62 & 0.7 & 3.33 & 185 \\
\hline
\end{tabular}


Table 3. Cont.

\begin{tabular}{|c|c|c|c|c|c|c|c|c|c|c|c|c|c|c|c|c|}
\hline Pyrite ID & $\mathbf{V}$ & Co & $\mathrm{Ni}$ & $\mathrm{Cu}$ & $\mathrm{Zn}$ & As & Se & Mo & Ag & $\mathrm{Sb}$ & $\mathrm{Te}$ & $\mathrm{Au}$ & $\mathrm{Tl}$ & $\mathrm{Pb}$ & Cd & $\mathbf{B i}$ \\
\hline Py 1_A2 & 3 & 412 & 145 & 79 & 5599 & 2532 & BDL & 32.63 & 1.2 & 58 & 0 & 0.41 & 0.38 & 26.1 & 26 & 27 \\
\hline Py 1_A3 & 6.7 & 2483 & 695 & 223 & 76 & 2655 & BDL & 61.23 & 8 & 164 & 35 & 0.43 & 1.72 & 0.6 & 7.57 & 153 \\
\hline Py 1_A4 & 0.5 & 5162 & 1557 & 973 & 1165 & 4555 & BDL & 80.24 & 13 & 227 & 60 & 0.28 & 0.85 & 9.7 & 10 & 198 \\
\hline Py 1_A5 & 2.8 & 1975 & 742 & 409 & 747 & 1992 & BDL & 54.4 & 8 & 21 & 24 & 0.41 & 0.78 & 0.2 & 3.6 & 215 \\
\hline Py 1_A6 & 1.7 & 3859 & 994 & 6317 & 30,925 & 3538 & 545 & 60.59 & 15 & 140 & 34 & 0.38 & 1.07 & 71.8 & 72 & 180 \\
\hline Py 1_A7 & 32.5 & 6558 & 2182 & 480 & 141 & 6485 & BDL & 82.49 & 16 & 209 & 43 & 1.29 & 0.97 & 0.1 & 4.07 & 234 \\
\hline Py 1_A8 & 0 & 578 & 153 & 114 & 515 & 568 & BDL & 47.29 & 1.6 & 157 & 0 & 0.52 & 0.48 & 0.1 & 4.57 & 15 \\
\hline Py 1_A9 & 3.2 & 3495 & 899 & 363 & 3725 & 3674 & BDL & 58.25 & 11 & 38 & 44 & 0.41 & 1.07 & 9.5 & 10 & 200 \\
\hline Py2_A & 20 & 1887 & 389 & 217 & 149 & 6111 & BDL & 12.86 & 4 & 349 & 63 & 0.29 & 2.85 & 620 & 2.02 & 295 \\
\hline Py2_B & 13.1 & 971 & 233 & 178 & 158 & 8819 & BDL & 6.38 & 2 & 154 & 30 & 0.11 & 1.24 & 413 & 1.83 & 160 \\
\hline Py2_C & 7.1 & 1452 & 265 & 380 & 85 & 5979 & BDL & 10.2 & 4 & 225 & 31 & 0.11 & 1.19 & 463 & 1.8 & 201 \\
\hline Py2_D & 7.5 & 1971 & 335 & 181 & 559 & 6189 & BDL & 5.61 & 4 & 303 & 55 & 0.11 & 1.53 & 682 & 1.8 & 312 \\
\hline Py2_E & 12.8 & 1510 & 258 & 174 & 100 & 5703 & BDL & 6.83 & 3 & 239 & 37 & 0.24 & 1.77 & 660 & 1.92 & 231 \\
\hline Py3_A & 2.6 & 1838 & 402 & 415 & 457 & 10,671 & BDL & 29.83 & 7 & 233 & 58 & 0.32 & 1.94 & 901 & 1.57 & 288 \\
\hline Py3_B & 1.4 & 333 & 234 & 121 & 540 & 562 & 51 & 35.19 & 2 & 49 & 10 & 0.12 & 0.18 & 341 & 1.83 & 32 \\
\hline Py3_C & 0.2 & 266 & 199 & 139 & 779 & 549 & 61 & 36.62 & 1 & 54 & 8 & 0.13 & 0.19 & 483 & 1.92 & 32 \\
\hline Py3_D & 0 & 271 & 193 & 132 & 752 & 520 & 80 & 40.1 & 1 & 39 & 6 & 0.06 & 0.07 & 382 & 0.735 & 27 \\
\hline Py3_E & 15.3 & 270 & 102 & 87 & 704 & 434 & 73 & 29.37 & 0.5 & 36 & 3.54 & 0.13 & 0.19 & 476 & 1.995 & 11 \\
\hline Py3_F & 10.5 & 259 & 137 & 40 & 394 & 700 & 53 & 19.35 & 0.5 & 28 & 3.5 & 0.13 & 0.19 & 232 & 1.97 & 6 \\
\hline Py3_G & 13.1 & 366 & 314 & 145 & 634 & 650 & 49 & 17.38 & 2 & 45 & 7 & 0.11 & 0.12 & 730 & 3 & 36 \\
\hline Py3_H & 0.5 & 1465 & 674 & 508 & 1301 & 1514 & 68 & 31.25 & 5 & 117 & 14 & 0.33 & 0.19 & 1245 & 1 & 95 \\
\hline Py3_I & 1.3 & 345 & 347 & 141 & 400 & 746 & 77 & 154.53 & 3 & 59 & 11 & 0.18 & 0.11 & 2812 & 1 & 64 \\
\hline Py3_J & 0.8 & 617 & 397 & 116 & 290 & 767 & 77 & 23.25 & 3 & 54 & 13 & 0.23 & 0.1255 & 0.248 & 1.6 & 51 \\
\hline Py3_K & 0 & 687 & 301 & 143 & 198 & 687 & 72 & 11.92 & 3 & 42 & 13 & 0.25 & 0.121 & 0.123 & 1.6 & 42 \\
\hline Py3_L & 0 & 625 & 801 & 275 & 1210 & 1029 & 66 & 33.31 & 3 & 65 & 11 & 0.18 & 0.18 & 913 & 8 & 54 \\
\hline Py3_M & 0 & 811 & 472 & 148 & 1146 & 914 & 56 & 64.53 & 5 & 66 & 13 & 0.17 & 0.19 & 1.01 & 1 & 63 \\
\hline Py3_N & 0.1 & 642 & 384 & 135 & 782 & 853 & 62 & 24.66 & 2 & 47 & 8 & 0.12 & 0.09 & 416 & 1 & 44 \\
\hline Py3_O & 2.9 & 239 & 349 & 115 & 535 & 493 & 57 & 15.85 & 2 & 44 & 7 & 0.11 & 0.15 & 505 & 1 & 35 \\
\hline Py4_A & 0 & 467 & 138 & 145 & 1117 & 1166 & 48 & 54.89 & 1 & 60 & 3.22 & 0.12 & 0.176 & 688 & 1.8 & 19 \\
\hline Py4_B & 0.1 & 357 & 105 & 99 & 903 & 1146 & 53 & 65.3 & 0.4 & 56 & 3.24 & 0.12 & 0.177 & 580 & 1.8 & 17 \\
\hline Py4_C & 0.4 & 366 & 129 & 69 & 220 & 423 & 55 & 6.71 & 1 & 32 & 3.46 & 0.13 & 0.1895 & 203 & 2 & 22 \\
\hline Py4_D & 9.4 & 135 & 888 & 60 & 368 & 565 & 51 & 8 & 2 & 53 & 4 & 0 & 0.02 & 563 & 0 & 26 \\
\hline Py4_E & 0.1 & 341 & 166 & 140 & 645 & 587 & 53 & 26.25 & 2 & 59 & 10 & 0.19 & 0.12 & 649 & 1 & 49 \\
\hline Py4_F & 1 & 408 & 580 & 90 & 436 & 646 & 80 & 21.62 & 2 & 46 & 9 & 0.11 & 0.1 & 756 & 2 & 33 \\
\hline Py4_G & 0.2 & 515 & 686 & 93 & 406 & 774 & 72 & 62.21 & 3 & 62 & 14 & 0.12 & 0.16 & 0.55 & 1 & 50 \\
\hline Py4_H & 20.2 & 1127 & 974 & 319 & 58 & 1970 & BDL & 23.08 & 4 & 139 & 25 & 0.53 & 1.02 & 1.837 & 1.835 & 122 \\
\hline Py4_II & 0.1 & 0 & 10.6 & 1.58 & 0.21 & 19.17 & 13.17 & 0.09 & 0 & 0.01 & 0.1 & 0 & 0.15 & 1.05 & 0 & 0 \\
\hline Py5_A & 5.6 & 125 & 20 & 9 & 0.715 & 102 & BDL & 1.1 & 0.3345 & 25 & 2.28 & 0.09 & 0.39 & 66 & 1.465 & 1 \\
\hline Py5_B & 11.5 & 68 & 25 & 20 & BDL & 74 & BDL & 3.26 & 0.358 & 46 & 2.435 & 0.1 & 0.87 & 79 & 1.57 & 2 \\
\hline Py5_C & 0.4 & 26 & 51 & 12 & BDL & 100 & 19 & 0.05 & 0 & 58 & 0.18 & 0.07 & 0.16 & 84 & 0.29 & 3 \\
\hline Py5_D & 1.2 & 26 & 257 & 24 & 3 & 97 & 20 & 0 & 0 & 23 & 1 & 0.01 & 0.11 & 0.3 & 0 & 3 \\
\hline Py5_E & 0.3 & 152 & 611 & BDL & BDL & 810 & BDL & 1.213 & 1.1 & 211 & 0 & 0.36 & 0.33 & 0.3 & 3.13 & 1 \\
\hline Py5_F & 2.9 & 37 & 45 & 35 & BDL & 201 & BDL & 1.2335 & 1.1 & 35 & 0 & 0.36 & 1.12 & 0.4 & 3.185 & 2 \\
\hline Py5_G & 9.2 & 55 & 312 & BDL & 3.3 & 488 & BDL & 1.403 & 1.2 & 3 & 0 & 0.41 & 0.3815 & 0.2 & 3.62 & 4 \\
\hline Py5_H & 2.3 & 666 & 2214.2 & 27.44 & 15.38 & 0.88 & 44.86 & 630.06 & 6.7 & 103.78 & 11.65 & 0.11 & 1.5 & 1004 & 0.1 & 83.98 \\
\hline Py5_I & 17.8 & 566 & 1081.5 & 115.99 & 18.57 & 1.98 & 33.46 & 0.52 & 3 & 70.96 & 3.64 & 0.04 & 1.59 & 511.6 & 0.05 & 9.76 \\
\hline Py5_J & 26.7 & 499 & 1394.2 & 12.37 & 7.81 & 1.03 & 37.99 & 5.73 & 5.8 & 56.63 & 9.02 & 0.08 & 1.69 & 679.9 & 0.03 & 75.45 \\
\hline Py5_K & 5.1 & 586 & 2504.9 & 253.38 & 3.59 & 1.65 & 42.16 & 346.54 & 13.4 & 114.71 & 11.56 & 0.09 & 2.36 & 1125.4 & 0.06 & 104.48 \\
\hline Py5_L & 1 & 608 & 1450 & 65.84 & 2 & 0.95 & 38.15 & 0.52 & 2 & 68.65 & 1.6 & 0.02 & 1.49 & 320 & 0 & 11.03 \\
\hline Py5_M & 14.5 & 2.7 & 65.4 & 12.79 & 0.69 & 28.86 & 12.23 & 2.52 & 0 & 1.46 & 0.14 & 0 & 0.98 & 11.2 & 0.16 & 0.01 \\
\hline Py5_N & 14.8 & 2 & 65.1 & 25.48 & 1.28 & 50.43 & 13.67 & 2.04 & 0.1 & 1.86 & 0.1 & 0 & 1.65 & 17.9 & 0.15 & 0.02 \\
\hline Py5_O & 5 & 2.4 & 199.5 & 16.33 & 1.17 & 60.08 & 11.74 & 2.42 & 0 & 1.51 & 0.09 & 0 & 0.85 & 11.5 & 0.16 & 0.03 \\
\hline Py5_P & 0.7 & 2.3 & 29 & 55.21 & 0.84 & 115.04 & 27.5 & 45.34 & 0 & 0.58 & 0.19 & 0.01 & 0.07 & 2.8 & 0.03 & 0 \\
\hline Py5_Q & 3.1 & 8.5 & 408.9 & 29.2 & 6.15 & 576.22 & 48.32 & 17.28 & 0.2 & 1.87 & 0 & 0 & 0.08 & 4.2 & 0.06 & 0.01 \\
\hline Py5_R & 23.5 & 1.8 & 24.4 & 34.28 & 2.34 & 57.32 & 13.21 & 3.27 & 0.1 & 2 & 0.51 & 0.01 & 1.15 & 20.5 & 0.24 & 0.03 \\
\hline Py5_S & 19.5 & 2 & 19.4 & 20.34 & 0.89 & 22.61 & 10.89 & 5.75 & 0.1 & 0.39 & 0.09 & 0 & 1.28 & 16.8 & 0.07 & 0.02 \\
\hline Py5_T & 9.3 & 2.7 & 36.9 & 25.38 & 1.15 & 31.81 & 9.26 & 3.36 & 0.1 & 0.75 & 0.09 & 0.01 & 1.14 & 13.7 & 0.1 & 0.03 \\
\hline Py5_U & 7 & 143 & 73 & 78 & BDL & 353 & BDL & 2.86 & 1 & 174 & 2.43 & 0.1 & 0.84 & 699 & 1.645 & 31 \\
\hline Py5_V & 0.5 & 124 & 95 & 93 & 1.925 & 539 & BDL & 0.495 & 1 & 184 & 2.275 & 0.09 & 0.94 & 784 & 1.535 & 24 \\
\hline Py6_A & 4.2 & 48 & 24 & 25 & 0.705 & 68 & BDL & 0.513 & 0.4 & 64 & 2.35 & 0.1 & 0.87 & 221 & 1.59 & 9 \\
\hline Py6_B & 0.3 & 39 & 22 & 45 & 2.545 & 208 & BDL & 0.4885 & 2 & 136 & 2.235 & 0.09 & 1.07 & 545 & 1.535 & 8 \\
\hline Py6_C & 4.8 & 62 & 92 & 73 & 1.875 & 143 & BDL & 0.4975 & 2 & 151 & 2.275 & 0.09 & 1.84 & 506 & 1.54 & 15 \\
\hline Py6_D & 1.9 & 100 & 46 & 9 & 1.685 & 93 & BDL & 0.434 & 0.3 & 25 & 2.22 & 0.09 & 0.1255 & 105 & 1.43 & 2 \\
\hline Py6_E & 0.9 & 49 & 50 & 22 & BDL & 117 & BDL & 1.69 & 0.3 & 77 & 2.36 & 0.1 & 2.44 & 174 & 1.52 & 1 \\
\hline Py6_F & 0 & 112 & 19 & 4.655 & 1.895 & 140 & BDL & 0.714 & 0.4 & 25 & 3.195 & 0.12 & 0.84 & 29 & 1.8 & 0 \\
\hline Py6_G & 2.3 & 12 & 42 & 24 & 2.1665 & 74 & BDL & 0.7085 & 0.4 & 72 & 3.17 & 0.12 & 1.67 & 90 & 1.79 & 17 \\
\hline Py6_H & 0.4 & 26 & 764 & 47 & BDL & 729 & 107 & 0.09 & 0 & 59 & 8 & 0 & 0.04 & 273 & 0 & 12 \\
\hline Py6_I & 2.1 & 61 & 57 & 117 & 11 & 1115 & 65 & 2.16 & 1 & 167 & 7 & 0.08 & 0.23 & 1423 & 0 & 34 \\
\hline Py6_J & 0 & 679 & 119 & 6 & 2 & 303 & 24 & 0.05 & 0 & 1 & 0 & 0 & 0.03 & 0.03 & 0 & 1 \\
\hline Py6_K & 0.2 & 127 & 147 & 20 & 30 & 204 & 5 & 5.02 & 0 & 55 & 0 & 0.03 & 0.16 & 0 & 0 & 3 \\
\hline Py6_L & 1.7 & 64 & 49 & 8 & 2 & 354 & 33 & 0.15 & 0 & 9 & 1 & 0 & 0.25 & 0.7 & 1 & 6 \\
\hline Py6_M & 5.6 & 507.9 & 991 & 106.81 & 2.57 & 1.1 & 32.27 & 0.17 & 2.3 & 27.36 & 1.44 & 0.01 & 1.84 & 172.1 & 0.06 & 7.36 \\
\hline Py6_N & 7 & 500.4 & 1187.9 & 120.09 & 2.51 & 2.34 & 25.43 & 0.09 & 3.3 & 211.02 & 1.72 & 0.02 & 7.95 & 324.4 & 0.04 & 5.03 \\
\hline Py6_O & 0.1 & 384.9 & 743.18 & 5.55 & 0.78 & 0 & 25.8 & 0.03 & 0.1 & 72.66 & 0.29 & 0 & 1.64 & 42.7 & 0 & 0.45 \\
\hline Py6_P & 8.2 & 498.5 & 1151.4 & 12.91 & 10.85 & 3.9 & 36.93 & 0.35 & 3.1 & 78.45 & 2.14 & 0.01 & 1.71 & 282.4 & 0.05 & 8.07 \\
\hline Py6_Q & 1.8 & 591.4 & 1363.3 & 4.71 & 1.65 & 1.04 & 45.16 & 0.04 & 0.5 & 24.95 & 0.28 & 0 & 0.82 & 80.7 & 0 & 1.03 \\
\hline
\end{tabular}



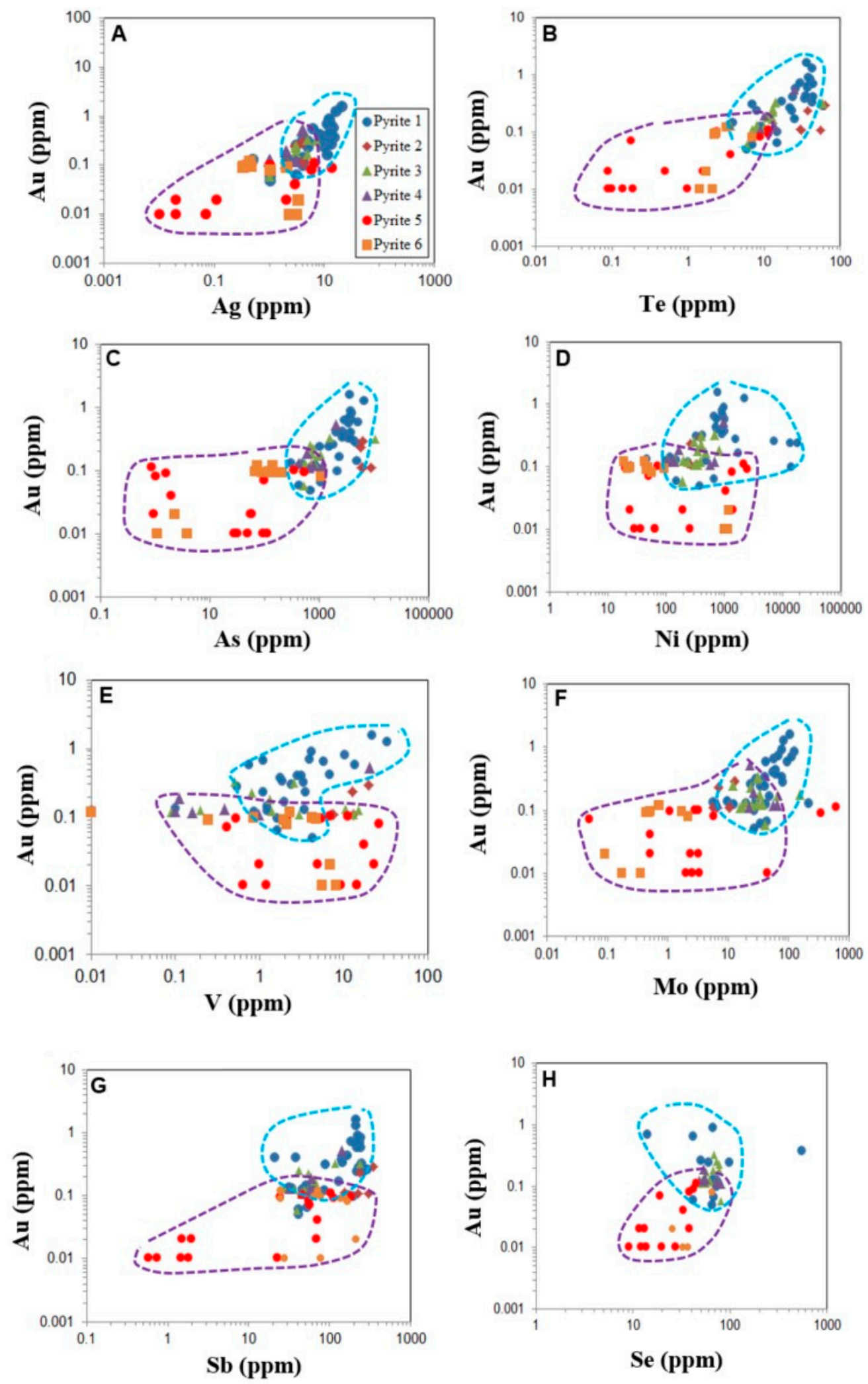

Late pyrite field gene field

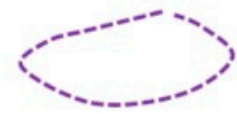

Early pyrite field

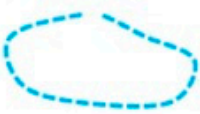

Figure 8. Scatter plot of Au versus other trace elements in pyrite from the Singa Formation, Malaysia. 
Table 4. Carbon and sulfur isotope composition from the black mudstones of Singa Formation, Malaysia.

\begin{tabular}{|c|c|c|c|c|c|c|}
\hline Sample & Formation & Weight & $\delta^{13} C_{\text {org }}$ & Corrected $\delta^{13} C_{\text {PDB }}$ & $\delta^{34} S$ & Source \\
\hline LA-3412 & Singa & 30.493 & -23.4 & -23.94 & -21.61 & Terrestrial \\
\hline LA-3512 & Singa & 21.0176 & -17.8 & -18.21 & -19.69 & Terrestrial \\
\hline LA-3612 & Singa & 14.8908 & -9.22 & -9.46 & -7.47 & Marine \\
\hline LA-3712 & Singa & 11.0084 & -11.31 & -11.59 & -8.44 & Marine \\
\hline LA-3812 & Singa & 7.9452 & -5.69 & -5.85 & 6.24 & Marine \\
\hline LA-3912 & Singa & 14.1708 & -8.17 & -8.38 & -4.19 & Marine \\
\hline LA-4012 & Singa & 24.8003 & -21.89 & -22.4 & -1.41 & Terrestrial \\
\hline LA-4112 & Singa & 22.6723 & -21.47 & -21.97 & -24.64 & Terrestrial \\
\hline LA-4212 & Singa & 30.0938 & -18.19 & -18.62 & -5.15 & Terrestrial \\
\hline LA-4312 & Singa & 29.3079 & -17.62 & -18.03 & 2.88 & Terrestrial \\
\hline LA-4412 & Singa & 28.1086 & -6.72 & -6.9 & -2.34 & Marine \\
\hline LA-5712 & Singa & 16.7783 & -9.2 & -9.44 & -13.86 & Marine \\
\hline
\end{tabular}

\section{Discussion}

\subsection{Gold Source Rock Characteristics}

It is known that strongly anomalous carbonaceous shales have gold content that ranges up to $42.5 \mathrm{ppb}[34,35]$. Reference [35] also documented a mean of $7 \mathrm{ppb} \mathrm{Au}$ from over 9000 analyses of black shales worldwide. The results indicate that gold content from the black mudstones, varying up to $62 \mathrm{ppb}$ (mean $58 \mathrm{ppb} \mathrm{Au}$ ), is well above the worldwide dataset. Additionally, the United States Geological Survey (USGS) standard black shale (SDO-1) has an As content of $68.5 \mathrm{ppm}$ [36]. Compared to these black mudstones, the As content varies from 6.7 to 55 ppm indicating a bit elevated As content for the SDO-1.

It is argued that organic carbon content in sedimentary source rocks varies from 0.2 to 20 wt.\% [37]. These rocks are enriched in $\mathrm{V}, \mathrm{As}, \mathrm{Mo}, \mathrm{Ni}, \mathrm{Ag}, \mathrm{Zn}, \mathrm{Cu}, \mathrm{Te}, \mathrm{Sb}, \mathrm{Pb}, \mathrm{Hg}, \mathrm{Bi}$, and $\mathrm{U}$. The $\mathrm{V}$ score $=\mathrm{V}+\mathrm{Mo}+\mathrm{Ni}+\mathrm{Zn}$ ranges from 250 to over $1000 \mathrm{ppm}$ making best shale source rocks with at least $5 \mathrm{ppb}$ gold, which may rise over $100 \mathrm{ppb}$ [37]. In these source rocks, As content ranges from 10 to over $100 \mathrm{ppm}$, with an approximate mean close to $30-50 \mathrm{ppm}$. Compared to mudstones in this study, the organic carbon content ranges up to $0.02 \mathrm{wt} . \%$ for the grey shales and varies between $0.1-0.7 \mathrm{wt} . \%$ for the black shales [37]. In this study, the V score is $256 \mathrm{ppm}$ for the Singa black mudstones, and a mean of $18 \mathrm{ppm}$ As. The V score of $256 \mathrm{ppm}$ is found within the range of best shale source rocks.

The evidence indicates that the black mudstones of the Singa Formation deposited during Late Devonian time right through the Carboniferous contain trace metals including gold that warrant further research work to fully assess its gold potential. Regionally, future research could also be carried out along the Sibumasu terrane from Indonesia to Myanmar.

In most sedimentary rocks, alumina $\left(\mathrm{Al}_{2} \mathrm{O}_{3}\right)$ can be used as a proxy for clay content in the sediments with low mobility during diagenesis [38-40]. It is documented that gold can be associated with clay minerals in the form of nanoparticles at the clay mineral grain rims [41]. Similarly, large-scale erosion and deposition of gold attached to clays in the Bendigo sedimentary basin has been documented [42]. Based on studies undertaken in the past, it has been demonstrated a sedimentary control on gold deposition into sedimentary basins. Previous research shows that alumina also plays a role in concentrating Sn by adsorption of $S n$ to clay particles, which is a common feature in sedimentary rocks [43]. Additionally, research on the Sn-W and U deposits in the Central European Variscan Belt (Bohemian Massif) had indicated that Sn mineralization was commonly associated with the clay mineral assemblage [44]. Therefore, correlation among alumina, organic carbon and trace elements can be used to assert controls on deposition of some trace elements into sedimentary basins. 
In this study, Pearson's coefficients of correlation are used to assess relationships between trace elements. Previous works have shown that elements such as $\mathrm{Mo}, \mathrm{V}, \mathrm{U}, \mathrm{Ni}, \mathrm{Cr}$, $\mathrm{As}$, and $\mathrm{Cu}$ can form organometallic complexes with humic acid found in organic matter and they get stuck in organic matter on the sea floor [3,39,45]. In the black mudstones of Singa Formation, the alumina content varies positively with $\mathrm{Ni}, \mathrm{Cu}, \mathrm{Zn}, \mathrm{As}$, and $\mathrm{Sb}$ and weakly with organic carbon (Table 5) implying that these trace metals may have been deposited attached to clay minerals or detrital input during deposition of the Singa Formation. The coefficients of correlation that are highlighted in red are 0.5 and above.

Table 5. Correlation coefficients between alumina, organic carbon, and trace elements in the black mudstones.

\begin{tabular}{|c|c|c|c|c|c|c|c|c|c|c|c|c|c|}
\hline Element & $\mathrm{Al}_{2} \mathrm{O}_{3}$ & Org.C & $S$ & $\mathbf{V}$ & $\mathrm{Cr}$ & $\mathrm{Ni}$ & $\mathrm{Cu}$ & $\mathrm{Zn}$ & As & Mo & Sn & $\mathrm{Sb}$ & $\mathbf{U}$ \\
\hline $\mathrm{Al}_{2} \mathrm{O}_{3}$ & 1 & & & & & & & & & & & & \\
\hline Org.C & 0.44 & 1 & & & & & & & & & & & \\
\hline S & -0.074 & -0.15 & 1 & & & & & & & & & & \\
\hline $\mathrm{V}$ & -0.11 & -0.62 & 0.25 & 1 & & & & & & & & & \\
\hline $\mathrm{Cr}$ & 0.35 & -0.1 & 0.62 & 0.48 & 1 & & & & & & & & \\
\hline $\mathrm{Ni}$ & 0.65 & 0.1 & 0.12 & 0.22 & 0.75 & 1 & & & & & & & \\
\hline $\mathrm{Cu}$ & 0.62 & 0.28 & 0.4 & 0.18 & 0.29 & 0.24 & 1 & & & & & & \\
\hline $\mathrm{Zn}$ & 0.89 & 0.34 & 0.11 & -0.17 & 0.49 & 0.75 & 0.45 & 1 & & & & & \\
\hline As & 0.66 & -0.0082 & -0.071 & 0.38 & 0.23 & 0.24 & 0.69 & 0.42 & 1 & & & & \\
\hline Mo & -0.21 & -0.31 & 0.79 & -0.048 & 0.24 & -0.11 & 0.24 & 0.044 & -0.15 & 1 & & & \\
\hline $\mathrm{Sn}$ & 0.025 & 0.37 & -0.63 & -0.14 & -0.63 & -0.48 & 0.15 & -0.31 & 0.38 & -0.55 & 1 & & \\
\hline $\mathrm{Sb}$ & 0.69 & 0.032 & -0.17 & 0.31 & 0.25 & 0.32 & 0.6 & 0.44 & 0.96 & -0.24 & 0.38 & 1 & \\
\hline $\mathrm{U}$ & 0.12 & -0.13 & 0.55 & 0.23 & 0.83 & 0.61 & -0.076 & 0.48 & -0.11 & 0.36 & -0.76 & -0.1 & 1 \\
\hline
\end{tabular}

Note: Numbers bold are equal to or greater than 0.5 .

Based on this study, there is a need to do more metallogenic research not only in Malaysia but also in neighboring countries such as Thailand, Indonesia, and Myanmar where glacial deposits crop out.

\subsection{Redox Conditions}

Total iron to aluminum $(\mathrm{Fe} / \mathrm{Al})$ and $\mathrm{Mo} / \mathrm{Al}$ ratios can be used to determine palaeoredox conditions [46-48]. It is argued that elevated Fe/ Al ratios indicates iron sulfide formation in the water column, and consequently the presence of dissolved sulfide. Therefore, $\mathrm{Fe} / \mathrm{Al}$ ratios can help distinguish conditions of ancient anoxia/suboxia and euxinia [47]. The values Mo/Al help differentiate oxic from anoxic water conditions in the ocean. Calculation of $\mathrm{Fe} / \mathrm{Al}$ and $\mathrm{Mo} / \mathrm{Al}$ ratios takes into consideration the following gravimetric conversion factors: (1) $\mathrm{Al}_{2} \mathrm{O}_{3}$ (wt.\%) content which is multiplied by 0.529 to get $\mathrm{Al}$ (wt.\%); and (2) $\mathrm{Fe}_{2} \mathrm{O}_{3}$ (wt.\%) content which is multiplied by 0.699 to obtain Fe (wt.\%). A similar approach was used in the Orca Basin, where the ratio Fe/Al values ranging from 0.40 to 0.50 indicated oxic conditions, whereas values from 0.55 to 0.75 were characteristic of anoxic zone [49].

Compared to this study, black mudstones have $\mathrm{Fe} / \mathrm{Al}$ ratio ranges from 0.09 to 0.77 (mean 0.5). Back mudstones $\mathrm{Mo} / \mathrm{Al}$ ratio fluctuates between 0.03 and 14.61. The two anomalous values for $\mathrm{Mo} / \mathrm{Al}$ ratio are indicative of low-oxygen environment indicating suboxic to oxic seafloor conditions. The organic carbon content of the Singa Formation is low (<0.5 wt.\%) suggesting that the mudstones may have been deposited in suboxic-oxic conditions. Low organic carbon and Mo, Ni, V contents indicate suboxic to oxic seawater conditions as higher organic carbon contents with elevated redox sensitive trace elements generally accumulate in anoxic seawater conditions [3]. In this study, mudstones have low contents in Mo (0.97-0.99 ppm), Ni (25-29 ppm), and V (130-171 ppm) indicating suboxicoxic seawater conditions. This study indicates that the mudstones containing clasts of glacial origin for the Singa Formation were not deposited during euxinic (environment devoid of enough oxygen or less aerated) marine conditions. However, some vanadium content and organic carbon indicates that there had been introduction of organic matter during deposition of silt and clay-size particles to form the mudstones. 


\subsection{Characterisation of Pyrite Generations}

The black mudstones contain early and late generations of pyrite: early generation which consists of framboids, and late generation made up of fine-grained euhedral pyrite clusters and coarse-grained euhedral pyrites. Framboidal pyrites often settle from the water column and grow within the sediments after deposition [6]. They reflect pore water composition, which is the exact copy of the sea water composition and commonly contained organic matter which may originate from land or sea. Pyrite framboids form during sediment compaction and transformation to sedimentary rocks which is called diagenesis. These pyrites are diagenetic and extract trace metals from the pore waters during growth [50]. However, syngenetic pyrites are smaller $(<5 \mu \mathrm{m}$ in diameter) generally contain $\mathrm{As}, \mathrm{Mo}$, and $\mathrm{Sb}$ and under euxinic conditions precipitates above the sediment seawater boundary directly from the seawater, thus reflecting a direct seawater composition [51]. Other previous works also indicated that framboids and micron-sized euhedral crystals of pyrite are generally early diagenetic pyrites [52].

Previous research has also documented that sedimentary pyrite can form syngenetically if the chemocline lies above the sediment-water interface, or diagenetically if the chemocline lies at or below the sediment-water interface [3]. The boundary in a body of water (also known as chemocline or cline), can form a layer caused by a strong, vertical chemistry gradient. Syngenetic pyrite consists of fine-grained, euhedral crystals $(<5 \mu \mathrm{m}$ in diameter); however, diagenetic pyrite is made up of larger ( $>10 \mu \mathrm{m}$ in diameter), spherical framboids $[3,53]$. Other researchers indicated that framboidal pyrites form during the early stage of diagenesis, with euhedral pyrite forming later in burial as dissolved sulfide concentrations decreased [54,55]. Based on the above literature knowledge, the textures of pyrites 1,2, and 3 are indicative of a diagenetic origin as they are mostly framboids and micro-euhedral particles [37]. In the early generations of pyrite (pyrites 1,2, and 3 ) in the black mudstones (this study), gold strongly correlates positively with $\mathrm{V}, \mathrm{Ag}$, and $\mathrm{Co}$ (Figure 8; Table 6). In addition, gold weakly correlates with $\mathrm{As}, \mathrm{Mo}, \mathrm{Sb}$, and $\mathrm{Te}$ (Table 6). In the late generations of pyrite (pyrites 4, 5, and 6), gold strongly correlates with $\mathrm{Cu}, \mathrm{As}, \mathrm{Cd}$, and $\mathrm{Te}$ and shows weak correlation with $\mathrm{Mn}, \mathrm{Co}$, $\mathrm{Se}$, and $\mathrm{Sb}$ (Table 7).

Table 6. Pearson coefficients of correlation of trace elements in early generations of pyrite.

\begin{tabular}{|c|c|c|c|c|c|c|c|c|c|c|c|c|c|c|c|c|}
\hline Element & V & Co & $\mathrm{Ni}$ & $\mathrm{Cu}$ & $\mathrm{Zn}$ & As & Se & Mo & Ag & $\mathrm{Cd}$ & $\mathrm{Sb}$ & $\mathrm{Te}$ & $\mathrm{Au}$ & $\mathrm{Tl}$ & $\mathrm{Pb}$ & Bi \\
\hline \multicolumn{17}{|l|}{$\mathrm{Mn}$} \\
\hline $\mathrm{V}$ & 1 & & & & & & & & & & & & & & & \\
\hline Co & 0.43 & 1 & & & & & & & & & & & & & & \\
\hline $\mathrm{Ni}$ & -0.083 & 0.054 & 1 & & & & & & & & & & & & & \\
\hline $\mathrm{Cu}$ & -0.0013 & 0.35 & -0.0013 & 1 & & & & & & & & & & & & \\
\hline $\mathrm{Zn}$ & -0.099 & 0.22 & 0.0098 & 0.56 & 1 & & & & & & & & & & & \\
\hline As & 0.45 & 0.52 & -0.087 & 0.12 & 0.041 & 1 & & & & & & & & & & \\
\hline $\mathrm{Se}$ & -0.042 & 0.49 & -0.0058 & 0.98 & 0.99 & 0.43 & 1 & & & & & & & & & \\
\hline Mo & 0.0044 & 0.35 & -0.058 & 0.051 & 0.045 & -0.027 & 0.04 & 1 & & & & & & & & \\
\hline $\mathrm{Ag}$ & 0.36 & 0.87 & 0.12 & 0.44 & 0.29 & 0.43 & 0.54 & 0.45 & 1 & & & & & & & \\
\hline $\mathrm{Cd}$ & -0.077 & 0.29 & 0.11 & 0.51 & 0.94 & 0.082 & 0.95 & 0.062 & 0.32 & 1 & & & & & & \\
\hline $\mathrm{Sb}$ & 0.35 & 0.57 & 0.35 & 0.12 & -0.0013 & 0.64 & 0.078 & 0.1 & 0.49 & 0.089 & 1 & & & & & \\
\hline $\mathrm{Te}$ & 0.4 & 0.72 & -0.018 & 0.2 & 0.11 & 0.78 & 0.35 & 0.16 & 0.65 & 0.18 & 0.77 & 1 & & & & \\
\hline $\mathrm{Au}$ & 0.56 & 0.75 & -0.019 & 0.076 & 0.012 & 0.35 & 0.13 & 0.44 & 0.82 & 0.023 & 0.43 & 0.49 & 1 & & & \\
\hline $\mathrm{Tl}$ & 0.26 & 0.57 & 0.22 & 0.32 & 0.029 & 0.63 & 0.08 & 0.13 & 0.55 & 0.1 & 0.81 & 0.78 & 0.4 & 1 & & \\
\hline $\mathrm{Pb}$ & -0.071 & 0.031 & 0.87 & 0.013 & -0.072 & -0.12 & -0.049 & 0.042 & 0.091 & 0.043 & 0.24 & -0.062 & -0.037 & 0.17 & 1 & \\
\hline $\mathrm{Bi}$ & 0.46 & 0.7 & -0.035 & 0.19 & 0.11 & 0.8 & 0.35 & 0.15 & 0.66 & 0.17 & 0.75 & 0.95 & 0.55 & 0.78 & -0.047 & 1 \\
\hline
\end{tabular}

Note: Numbers bold are equal to or greater than 0.5 .

Correlation matrix (Tables 6 and 7) shows that Au has strong affinity with $\mathrm{V}, \mathrm{Ag}$ and Co in the sedimentary pyrite which means these trace elements and gold may have been introduced into the sediments at the same time. Elements such as As and Te which do not have strong relationship with $\mathrm{Au}$ in the early pyrite generations may have been brought into the mudstones during circulation of metamorphic-hydrothermal fluids together with gold.

Compared to early pyrites (pyrite 1, 2, and 3), the later pyrites (pyrite 4, 5, and 6) have the following ratio limits: $\mathrm{Co} / \mathrm{Ni}(0.01-6), \mathrm{Zn} / \mathrm{Ni}(0.001-9), \mathrm{Cu} / \mathrm{Ni}(0.003-2), \mathrm{As} / \mathrm{Ni}$ (0.0008-20), Te/Au (3-214), As/Au (>8), Sb/Au (>58), and Bi/Au (>1). Comparatively, these geochemical values are much lower relative to those of diagenetic pyrites [33]. There- 
fore, the textures of pyrite 4, 5, and 6 combined with their geochemical ratio values suggest hydrothermal-metamorphic fluids were involved during pyrite formation. The fact that all the pyrites plot on or below the line $\mathrm{Ag} / \mathrm{Au}=1$ strongly suggests that pyrites 4,5 , and 6 are metamorphic rather than hydrothermal $[33,42]$ and formed by the recrystallization of early pyrite generations (pyrites 1,2, and 3). The presence of pyrrhotite associated with pyrite strongly indicates that some greenschist facies conditions were prevalent over post-lithification of the Singa Formation, pointing to circulation of metamorphic fluids. Such conditions may cause remobilization of As and Au during metamorphism [56].

Table 7. Pearson coefficients of correlation of trace elements in late generations of pyrite.

\begin{tabular}{|c|c|c|c|c|c|c|c|c|c|c|c|c|c|c|c|c|}
\hline Element & V & Co & $\mathrm{Ni}$ & $\mathrm{Cu}$ & $\mathrm{Zn}$ & As & Se & Mo & $\mathrm{Ag}$ & $\mathrm{Cd}$ & $\mathrm{Sb}$ & $\mathrm{Te}$ & $\mathrm{Au}$ & $\mathrm{Tl}$ & $\mathrm{Pb}$ & Bi \\
\hline \multicolumn{17}{|l|}{$\mathrm{Mn}$} \\
\hline $\mathrm{V}$ & 1 & & & & & & & & & & & & & & & \\
\hline Co & 0.12 & 1 & & & & & & & & & & & & & & \\
\hline $\mathrm{Ni}$ & 0.12 & 0.74 & 1 & & & & & & & & & & & & & \\
\hline $\mathrm{Cu}$ & 0.082 & 0.63 & 0.36 & 1 & & & & & & & & & & & & \\
\hline $\mathrm{Zn}$ & -0.28 & 0.2 & -0.13 & 0.31 & 1 & & & & & & & & & & & \\
\hline As & -0.087 & 0.35 & -0.11 & 0.64 & 0.59 & 1 & & & & & & & & & & \\
\hline Se & -0.46 & 0.31 & 0.21 & 0.35 & 0.53 & 0.67 & 1 & & & & & & & & & \\
\hline Mo & -0.11 & 0.36 & 0.62 & 0.21 & 0.029 & -0.057 & 0.11 & 1 & & & & & & & & \\
\hline $\mathrm{Ag}$ & 0.16 & 0.61 & 0.82 & 0.57 & -0.047 & -0.0046 & 0.16 & 0.67 & 1 & & & & & & & \\
\hline $\mathrm{Cd}$ & -0.28 & -0.024 & -0.46 & 0.16 & 0.41 & 0.41 & 0.4 & -0.15 & -0.17 & 1 & & & & & & \\
\hline $\mathrm{Sb}$ & -0.14 & 0.3 & 0.25 & 0.49 & -0.036 & 0.29 & 0.34 & 0.16 & 0.38 & 0.19 & 1 & & & & & \\
\hline $\mathrm{Te}$ & 0.13 & 0.66 & 0.44 & 0.71 & 0.2 & 0.64 & 0.69 & 0.39 & 0.58 & 0.2 & 0.34 & 1 & & & & \\
\hline $\mathrm{Au}$ & 0.08 & 0.5 & 0.013 & 0.66 & 0.24 & 0.69 & 0.44 & 0.12 & 0.24 & 0.6 & 0.35 & 0.78 & 1 & & & \\
\hline $\mathrm{Tl}$ & 0.21 & 0.23 & 0.36 & 0.14 & -0.31 & -0.33 & -0.3 & 0.076 & 0.32 & -0.23 & 0.46 & -0.078 & -0.11 & 1 & & \\
\hline $\mathrm{Pb}$ & -0.15 & 0.23 & 0.36 & 0.41 & 0.27 & 0.26 & 0.47 & 0.45 & 0.55 & 0.011 & 0.61 & 0.32 & 0.12 & 0.016 & 1 & \\
\hline $\mathrm{Bi}$ & 0.19 & 0.67 & 0.59 & 0.71 & 0.13 & 0.47 & 0.4 & 0.57 & 0.79 & 0.088 & 0.41 & 0.9 & 0.68 & 0.0083 & 0.51 & 1 \\
\hline
\end{tabular}

Note: Numbers bold are equal to or greater than 0.5 .

\subsection{Trace Element Deportment in Pyrite}

The early pyrite generation (pyrites 1,2 , and 3 ) is enriched in most trace elements except $\mathrm{Tl}$ compared to late pyrite generation (pyrites 4,5 , and 6 ). This feature is commonly observed in metamorphic pyrites formed by recrystallization of sedimentary pyrites $[42,57]$.

It is documented in previous works, that the arsenic concentration of hydrothermal pyrite controls its ability to retain gold within the pyrite structure [58,59]. The relationship is that the more arsenic is there in pyrite, the more gold is found. The authors of [31] argued that the same relationship Au-As applies for diagenetic pyrite in black shales. The $\mathrm{Au}-\mathrm{As}$ relationship for all pyrites displaying the gold saturation line for arsenian pyrite is shown in Figure 9. The gold saturation line represents the solubility limit of solid solution of $\mathrm{Au}$ in relation to As for most temperatures that range from $150{ }^{\circ} \mathrm{C}$ to $250{ }^{\circ} \mathrm{C}$. Pyrite analyses show $\mathrm{Au}-\mathrm{As}$ contents plot below the gold saturation line. The evidence indicates that most gold is locked up or refractory in the pyrite structure or structurally trapped in solid solution (in the chemical state of $\mathrm{Au}^{+1}$ ). It also indicates that gold is not existing in the form of nanoparticles (in the chemical form of $\mathrm{Au}^{0}$ ) in pyrite. Au-As relationship (in Figure 9) reveals that sedimentary pyrites have elevated Au and As content which drops in metamorphic-hydrothermal pyrites indicative of loss of these trace elements in metamorphic fluid flows.

\subsection{Sulfur and Carbon Isotope Compositions}

Research by [60] documented that sulfur of mantle and magmatic origin has $\delta^{34} \mathrm{~S}$ values close to zero relative to the standard (CDT). In contrast, $\delta^{34} \mathrm{~S}$ values of approximately +20 per mil imply that marine evaporates, or seawater is likely to be the source of the sulfur. Seawater sulfates can yield variable heavy $\delta^{34} S$ isotopic values varying from +10 to $+35 \%$ from the Cambrian to the present [61]. Modern seawater sulfates have $\delta^{34} S$ values, which range from +18 to $20 \%$ [61], whereas sedimentary environments can have variable $\delta^{34} \mathrm{~S}$ values ranging from -70 to $>+20 \%$ [62]. Furthermore, sulfides in hydrothermal systems 
mostly yield $\delta^{34} \mathrm{~S}$ values around $0.0 \%$ with values ranging up to $10 \%$ in certain conditions of temperature and $\mathrm{pH}$, as well as the preserved primary isotopic compositions [62].

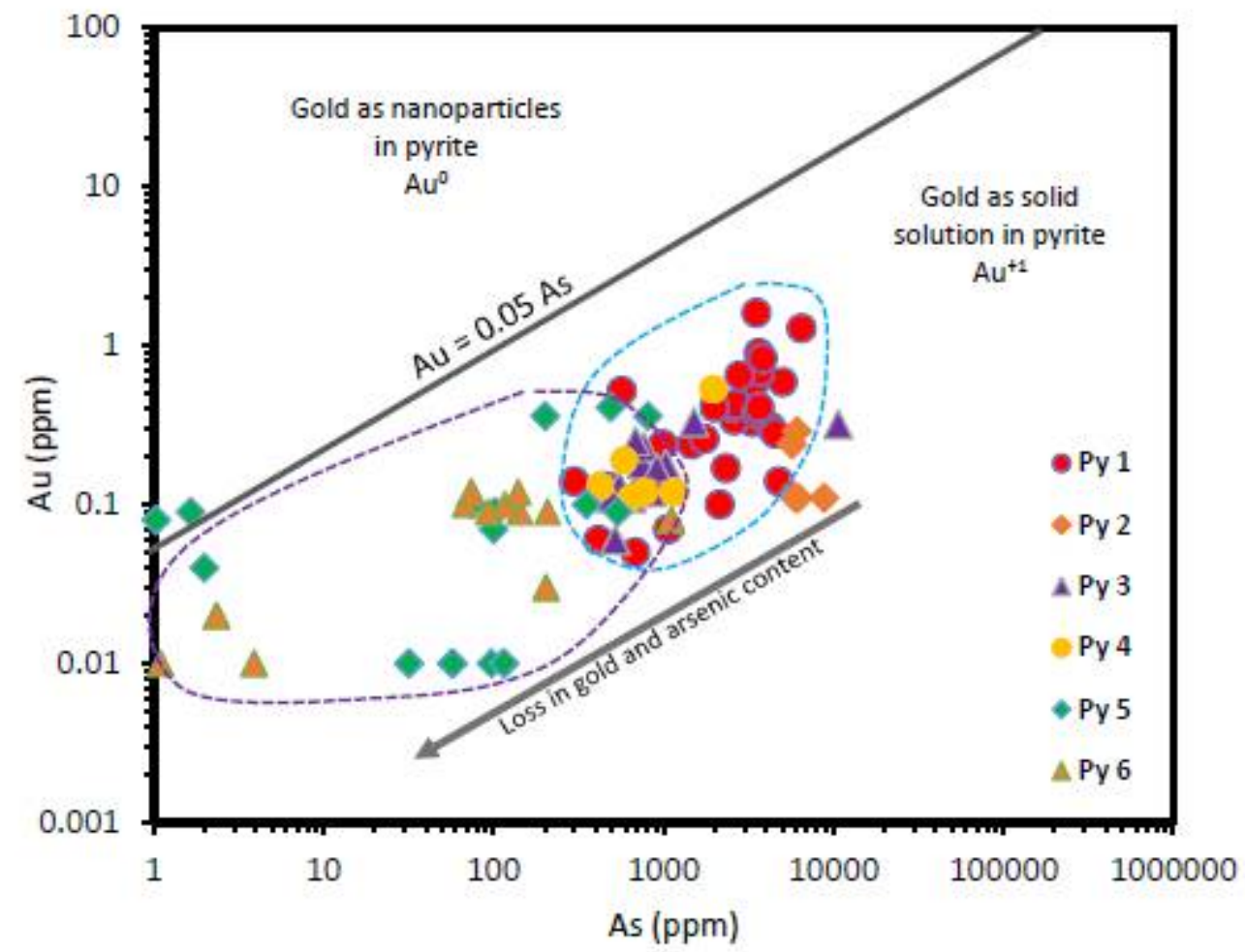

Figure 9. $\mathrm{Au}-\mathrm{As}$ relationship in pyrite from the black mudstones, Langkawi, Malaysia. Au $=0.05 \mathrm{As}$ is the gold saturation line for arsenian pyrite from Large et al. (2011).

Sulfur can also be introduced by metamorphic fluids from wall rocks $[63,64]$ or from the deep crust during amphibolite-grade and granulite-grade metamorphism [65]. The $\delta^{34} \mathrm{~S}$ values from the Anatahan eruptions showed a range between $-0.5 \%$ ond $+2.5 \%$ [ [66] . These authors also indicated $\delta^{34} \mathrm{~S}$ values from the melts varying from $0 \%$ to $+2 \%$; however, the $\delta^{34} S$ values from the sulfates contained in the ash range between $+2.5 \%$ and $+3 \%$. Moreover, most $\delta^{34} S$ values of sulfides from skarn deposits are between -5 per mil and +8 per mil indicative of a magmatic origin of the sulfur [67]. Compared to this study, sulfur may have derived from a sedimentary and magmatic source. Therefore, these glacier-derived, pebbly mudstones of the Singa Formation contain sulfur that originated from pre-existing underlying rocks. These rocks were eroded during glacial processes and resulted in the accumulation of fine-grained sediments forming the Singa Formation mudstones in the marine environment The evidence of marine deposition is both supported by the presence of brachiopods in the mudstones and organic carbon of marine origin (Table 4).

Based on the findings in this study, values of $\delta^{34} \mathrm{~S}$ that range between $-1.4 \%$ and $+2.8 \%$ being closer to $0 \%$ suggest that sulfur probably originated from igneous or magmatic source. Other negative and positive values within the $\delta^{34} \mathrm{~S}$ range $(-24.6 \%$ to $+6.2 \%$ o in this study, probably attribute pyrite formation to microbial sulfate reduction pointing to biogenic activity. Recent research work conducted on the Dalradian Supergroup Neoproterozoic of Britain and Ireland returned sulfur isotope values of $-31.6 \%,+17.1 \%$ and $-4.6 \%$ interpreted that sulfur derived from sea water sulfate reduction [68].

Commonly, $\delta^{13} \mathrm{C}_{\text {org }}$ (organic carbon isotopic composition) for the marine plants ranges from $-8 \%$ to $-17 \%$; however, for the land plants, it is between $-22 \%$ ond $-29 \%$ [69-72]. Compared to previous research, the Singa Formation has $\delta^{13} \mathrm{C}_{\text {org }}$ values that range from $-23.9 \%$ to $-5.8 \%$ o (Table 4 ) indicating a mixed, land-derived, and marine source of organic carbon. 


\section{Conclusions}

Mudstones of the Singa Formation contain lonestones, which are indicative of glacial origin for these rocks. This study has shown that the mudstones deposited under suboxicoxic conditions in marine environment. Findings in this study show that the gold content in the mudstones range up to $62 \mathrm{ppb}$, which is higher than the crustal level, making them gold source rocks. This study has shown early enrichment of gold and other trace elements in sedimentary pyrites found in the pebbly mudstones. The late generations of pyrite which include the metamorphic-hydrothermal pyrites (pyrites 4, 5, and 6) with low trace element content are associated with pyrrhotite suggesting that trace elements and gold may have been liberated from early pyrite generations (pyrites 1,2, and 3) and transported in metamorphic-hydrothermal fluids. The strong correlation among $\mathrm{Al}_{2} \mathrm{O}_{3}$ and trace elements such as $\mathrm{Ni}, \mathrm{Cu}, \mathrm{Zn}, \mathrm{As}$, and $\mathrm{Sb}$ probably indicate that these elements were transported attached to clay particles during sedimentation. The $\delta^{13} \mathrm{C}_{\text {org }}$ isotopic response indicates a mixed, land-derived, and marine source of organic carbon. The $\delta^{34} S$ isotopic signature for pyrite likely indicates magmatic and microbial sulfate reduction linked to biogenic activity.

Author Contributions: For this research Article, author individual contributions are the following: Conceptualization, C.M., R.R.L. and K.Z.; methodology, C.M., E.L.; software, C.M.; validation, K.Z., R.R.L. and C.M.; formal analysis, C.M.; investigation, M.S.L., K.R.M.; resources, Z.E.; data curation, C.M.; writing-original draft preparation, C.M.; writing-review and editing, C.M., E.L.; visualization, C.M., Z.E. and M.B.I.B.; supervision, R.R.L. and K.Z.; project administration, K.Z.; funding acquisition, K.Z., R.R.L. All authors have read and agreed to the published version of the manuscript.

Funding: This research was funded by University of Tasmania-APA Scholarship and the Southeast Asia Ore Deposit Research Project.

Data Availability Statement: Not applicable.

Acknowledgments: The authors would like to thank for the huge support from the Project entitled "Ore Deposits of SE Asia Project" and the University of Tasmania-APA (Australian Postgraduate Award) Scholarship. Many thanks also go to Jay Thompson, and Elena Lounejeva for their assistance on X-ray fluorescence analysis at CODES geochemical laboratory, University of Tasmania, Australia. This paper is part of the first author Doctoral $(\mathrm{PhD})$ research project undertaken at the Centre for Ore Deposits and Earth Sciences (CODES), University of Tasmania, Australia.

Conflicts of Interest: The authors declare no conflict of interest.

\section{References}

1. Pašava, J.; Sulovsky, P.; Kovalova, M. Geochemistry and mineralogy of Proterozoic metal-rich black shales from the Bohemian massif Czech Republic with a description of possible new Molybdenum selenide and Telluride phases. Can. Mineral. 1993, 31, 745-754.

2. Rimmer, S.M. Geochemical paleoredox indicators in Devonian-Mississippian black shales, Central Appalachian Basin (USA) Department of Geological Sciences, University of Kentucky, Lexington, KY 40506-0053, USA. Chem. Geol. 2004, 206, 373-391. [CrossRef]

3. Tribovillard, N.; Algeo, T.J.; Lyons, T.; Riboulleau, A. Trace metals as paleoredox and paleoproductivity proxies: An update. Chem. Geol. 2006, 232, 12-32. [CrossRef]

4. Pi, D.H.; Cong-Qiang, A.G.L.; Zhoud, S.; Shao-Yong, J. Trace and rare earth element geochemistry of black shale and kerogen in the early Cambrian Niutitang Formation in Guizhou province, South China: Constraints for redox environments and origin of metal enrichments. Precambrian Res. 2013, 225, 218-229. [CrossRef]

5. Bloomstein, E.I.; Clark, J.B. Geochemistry of the Ordovician High-Calcium Black Shales Hosting Major Gold Deposits of the Getchell Trend in Nevada; U.S. Geological Survey Circular: Reston, VA, USA, 1991; 1058, pp. 1-5.

6. Makoundi, C.; Khin Zaw Large, R.R.; Meffre, S.; Chun-Kit, L.; Guan, H.T. Geology, geochemistry and metallogenesis of the Selinsing gold deposit, Central Malaysia. Gondwana Res. 2014, 26, 241-261. [CrossRef]

7. Endut, Z.; Tham Fatt, N.G.; Abdul Aziz, J.H.; Meffre, S.; Makoundi, C. Characterization of Galena and Vein Paragenesis in the Penjom Gold Mine, Malaysia: Trace Elements, Lead Isotope Study and Relationship to Gold Mineralization Episodes. Acta Geol. Sin. 2015, 89, 1801-1840.

8. Makoundi, C. Geochemistry of Black Shales, Sandstones, and Chert in Malaysia: Insights into Gold Source Rocks. Ph.D. Thesis, University of Tasmania, Hobart, Australia, 2016; 383p. 
9. Cromie, P.; Makoundi, C.; Zaw, K.; Cooke, D.R.; White, N.; Ryan, C. Geochemistry of Au-bearing pyrite from the Sepon Mineral District, Laos DPR, Southeast Asia: Implications for ore genesis. J. Asian Earth Sci. 2018, 164, 194-218. [CrossRef]

10. Stauffer, P.H.; Lee, C.P. Late Palaeozoic glacial marine facies in Southeast Asia and its implications. Bull. Geol. Soc. Malays. 1986, 20, 363-397. [CrossRef]

11. Ali, C.A.; Mohamed, K.R.; Leman, M.S.; Komoo, I.; Unjah, T. Field Guide to Geology of Langkawi Geopark; Institut Alam Sekitar dan Pembangunan, Universiti Kebangsaan Malaysia: Selangor, Malaysia, 2008; pp. 1-82. ISBN 9839444891/9789839444896.

12. Ali, C.A.; Mohamed, K.R.; Leman, M.S.; Komoo, I.; Tanut Cocks, L.R.M.; Fortey, R.A.; Lee, C.P. A Review of Lower and Middle Palaeozoic biostratigraphy in west Peninsular Malaysia and southern Thailand in its context within Sibumasu terrane. J. Asian Earth Sci. 2005, 24, 703-717.

13. Liew, T.C.; McCulloch, M.T. Genesis of granitoid batholiths of Peninsular Malaysia and implications for models of crustal evolution: Evidence from Nd-Sr isotopic and U-Pb zircon study. Geochim. Cosmochim. Acta 1985, 49, 587-600. [CrossRef]

14. Jones, C.R. Lower Paleozoic. In Geology of the Malay Peninsula; Gobbett, D.J., Hutchison, C.S., Eds.; Wiley-Interscience: New York, NY, USA, 1973; pp. 25-60.

15. Ong, S.S. Geology of the Muda Dam Area, Kedah, West Malaysia. Bachelor's Thesis, University of Malaya, Kuala Lumpur, Malaysia, 1969.

16. Metcalfe, I. Tectonic evolution of the Malay Peninsula. J. Asian Earth Sci. 2013, 76, 195-213. [CrossRef]

17. Metcalfe, I. Tectonic evolution of Sundaland. Bull. Geol. Soc. Malays. 2017, 63, 27-60. [CrossRef]

18. Leman, M.S.; Yop, A. Early Permian sequence from Sungai Itau quarry, Langkawi: Its age, depositional environment and paleoclimatic implication. Geol. Soc. Malays. 2002, 46, 163-170.

19. Basir, J.; Harun, Z.; Hassan, S.N. Black siliceous deposits in Peninsular Malaysia: Their occurrence and significance. Bull. Geol. Soc. Malays. 2003, 46, 149-154. [CrossRef]

20. Mitchell, A.H.G.; Young, B.; Jantaranipa, W. The Phuket group, Peninsular Thailand: A Palaeozoic Geosynclinal deposit. Geol. Mag. 1970, 107, 411-428. [CrossRef]

21. Waterhouse, J.B. An early Permian cool-water fauna from pebbly mudstones in south Thailand. Geol. Mag. 1982, 119, 337-354. [CrossRef]

22. Allman, R.; Crocket, J.H. Handbook of Geochemistry; Wedepohl, K.H., Ed.; Springer: New York, NY, USA, 1978 ; p. 79.

23. Boyle, R.W. The geochemistry of gold and its deposits. Geol. Surv. Can. Bull. 1979, 280, 584p.

24. Shawe, D.R. Geology and Resources of Gold in the United States; U.S. Government Printing Office: Washington, DC, USA, 1988; pp. A1-A8.

25. Krom, M.D.; Berner, R.A. A rapid method for the determination of organic and carbonate carbon in geological samples. J. Sediment. Res. 1983, 53, 600-663. [CrossRef]

26. Danyushevsky, L.V.; Robinson, P.; Gilbert, S.; Norman, M.; Large, R.R.; McGoldrick, P.; Shelley, J.M.G. Routine quantitative multi-element analysis of sulfide minerals by laser ablation ICP-MS: Standard development and consideration of matrix effects. Geochem. Explor. Environ. Anal. 2011, 11, 51-60. [CrossRef]

27. Belousov, I. New Calibration Standard for LA-ICPMS Analysis of Sulphides. AGU, 2014 Fall Meeting. 2014. Available online: https://agu.confex.com/agu/fm14/webprogram/Paper19453.html (accessed on 5 April 2021).

28. Belousov, I.A.; Danyushevsky, L.V.; Olin, P.H.; Gilbert, S.E.; Thompson, J. STDGL3: A new calibration standard for sulphide analysis by LAICP-MS. In Proceedings of the 25th Goldschmidt Conference, Prague, Czech Republic, 16-21 August 2015.

29. Jochum, K.P. Reference Materials in Geochemical and Environmental Research. Analytical Geochemistry/Inorganic Instrumental Analysis, 2nd ed.; Elsevier Ltd.: Amsterdam, The Netherlands, 2014; Volume 15. [CrossRef]

30. Gilbert, S.E.; Danyushevsky, L.V.; Rodemann, T.; Shimizu, N.; Gurenko, A.; Meffre, S.; Thomas, H. Optimisation of laser parameters for the analysis of sulphur isotopes in sulphide minerals by laser ablation ICP-MS. J. Anal. At. Spectrom. 2014, $29,1042$. [CrossRef]

31. Large, R.R.; Danyushevsky, L.; Hollit, C.; Maslennikov, V.; Meffre, S.; Gilbert, S.; Bull, S.; Bull, S.; Scott, R.; Emsbo, P.; et al. Gold and Trace element zonation in pyrite using a laser imaging technique: Implications for the timing of gold in orogenic and Carlin-style sediment-hosted deposits. Econ. Geol. 2009, 104, 635-668. [CrossRef]

32. Robinson, B.W.; Kusakabe, M. Quantitative preparation of $\mathrm{SO} 2$ for $34 \mathrm{~S} / 32 \mathrm{~S}$ analyses from sulphides by combustion. Anal. Chem. 1975, 47, 1179-1181. [CrossRef]

33. Gregory, D.; Large, R.R.; Halpin, J.A.; Baturina, E.L.; Lyons, T.W.; Wu, S.; Sack, P.J.; Chappaz, A.; Maslennikov, V.V.; Bull, S.W. Trace element content of background sedimentary pyrite in black shales. Econ. Geol. 2015, 110, 1389-1410. [CrossRef]

34. Crocket, J.H. Distribution of gold in the Earth's crust. In Gold Metallogeny and Exploration; Foster, R.P., Ed.; Chapman and Hall: London, UK; New York, NY, USA, 1991; pp. 1-36.

35. Ketris, M.P.; Yudovitch, Y.E. Estimations of Clarkes for Carbonaceous bioliths: World averages for trace element contents in black shales and coals. Int. J. Coal Geol. 2009, 78, 135-148. [CrossRef]

36. Huyck, H.L.O. When is a metalliferous black shale not a black shale? U.S. Geol. Survev Circ. 1989, 1058, 42-56.

37. Large, R.R.; Bull, S.W.; Maslennikov, V.V. A carbonaceous sedimentary source-rock model for Carlin-type and orogenic gold deposits. Soc. Econ. Geol. 2011, 106, 331-358. [CrossRef]

38. Brumsack, H.J. Geochemistry of recent TOC-rich sediments from the Gulf of California and Black Sea. Geol. Rundsch. 1989, 78, 851-882. [CrossRef] 
39. Calvert, S.E.; Pedersen, T.F. Geochemistry of recent oxic and anoxic marine sediments: Implications for the geological record. Mar. Geol. 1993, 113, 67-88. [CrossRef]

40. Piper, D.Z.; Perkins, R.B. A modern vs. Permian black shales-the hydrography, primary, productivity and water-column chemistry of deposition. Chem. Geol. 2004, 206, 177-197. [CrossRef]

41. Hanlie, H.; Qinyan, W.; Jiampin, C. Occurrence and distribution of invisible gold in the Shewushan supergene gold deposit, Southeastern Hubei, China. Can. Mineral. 1999, 37, 1525-1531.

42. Thomas, H.V.; Large, R.R.; Bull, S.W.; Maslennikov, V.; Berry, R.F.; Fraser, R.; Froud, S.; Moye, R. Pyrite and pyrrhotite textures and composition in sediments, laminated quartz veins and reefs, at Bendigo Gold Mine, Australia; Insights for orogenesis. Econ. Geol. 2011, 106, 1-31. [CrossRef]

43. Slingerland, R.; Smith, N. Occurrence, and formation of water-laid placers. Annu. Rev. Earth Planet. Sci. 1986, 14, 113-147. [CrossRef]

44. René, M. Distribution and Origin of Clay Minerals during Hydrothermal Alteration of Ore Deposits. In Clay Minerals in NatureTheir Characterization, Modification and Application; Valášková, M., Martynkova, S.G., Eds.; InTech: Libchavy, Czech Republic, 2012; pp. 81-100. [CrossRef]

45. Wood, S.A. The role of humic substances in the transport and fixation of metals of economic interest (Au, Pt, Pd, U, V). Ore Geol. Rev. 1996, 11, 1-33. [CrossRef]

46. Sageman, B.B.; Lyons, T.W. Geochemistry of fine-grained sediments and sedimentary rocks. In Sediments, Diagenesis, and Sedimentary Rocks, Treatise on Geochemistry; MacKenzie, F., Ed.; Elsevier Publication: New York, NY, USA, 2004; Volume 7, pp. $115-158$.

47. Clarkson, M.O.; Wood, R.A.; Poulton, S.W.; Richoz Newton, R.J.; Kasemann, S.A.; Bowyer, F.; Krystyn, L. Dynamic anoxic ferruginous conditions during the end-Permian mass extinction and recovery. Nat. Commun. 2016, 7, 1-9. [CrossRef]

48. Raiswell, R.; Hardisty, D.S.; Lyons, T.W.; Canfield, D.E.; Owens, J.D.; Planavsky, N.J.; Poulton, S.W.; Reinhard, C.T. The iron paleoredox proxies: A guide to the pitfalls, problems, and proper practice. Am. J. Sci. 2018, 318, 491-526. [CrossRef]

49. Taylor, S.R.; McLennan, S.M. The Continental Crust: Its Composition and Evolution; Blackwell Malden Mass: Malden, MA, USA, $1985 ;$ p. 312.

50. Huerta-Diaz, M.A.; Morse, J.W. A quantitative method for determination of trace metal concentrations in sedimentary pyrite. Mar. Chem. 1990, 29, 119-144. [CrossRef]

51. Large, R.R.; Halpin, J.A.; Danyushevsky, L.V.; Maslennikov, V.V.; Bull, S.W.; Long, J.A.; Gregory, D.D.; Elena Lounejeva Lyons, T.W.; Sack, P.J.; McGoldrick, P.J.; et al. Trace element content of sedimentary pyrite as a new proxy for deep-time ocean-atmosphere evolution. Earth Planet. Sci. Lett. 2014, 389, 209-220. [CrossRef]

52. Cavalazzi, B.; Agangi, A.; Barbieri, R.; Gasparotto, G. The formation of low-temperature sedimentary pyrite and its relationship with biologically induced processes. Geol. Ore Depos. 2014, 56, 395-408. [CrossRef]

53. Wilkin, R.T.; Barnes, H.L.; Brantley, S.L. The size distribution of framboidal pyrite in modern sediments: An indicator of redox conditions. Geochim. Cosmochim. Acta 1996, 60, 3897-3912. [CrossRef]

54. Raiswell, R. Pyrite texture, isotopic, composition and the availability of iron. Am. J. Sci. 1982, 82, 1244-1263. [CrossRef]

55. Coleman, M.; Raiswell, R. Source of carbonate and origin of zonation in pyritiferous carbonate concretions: Evaluation of a dynamic model. Am. J. Sci. 1995, 295, 282-308. [CrossRef]

56. Large, R.R. Helen Thomas, Dave Craw, Anicia Henne and Simon Henderson. Diagenetic pyrite as a source for metals in orogenic gold deposits, Otago Schist, New Zealand. N. Z. J. Geol. Geophys. 2012, 55, 137-149. [CrossRef]

57. Large, R.R.; Maslennikov, V.V.; Robert, F.; Danyushevsky, L.V.; Chang, Z. Multistage sedimentary and metamorphic origin of pyrite and gold in the Giant Sukhoi log deposit, Lena Gold Province, Russia. Econ. Geol. 2007, 102, 1233-1267. [CrossRef]

58. Fleet, M.E.; Chryssoulis, S.L.; MacLean, P.J.; Davidson, R.; Weisener, C.G. Arsenian pyrite fom gold deposits: Au and As distribution investigated by SIMS and EMF, and color staining and surface oxidation by XPS and LIMS. Can. Mineral. 1993, 31, 1-17.

59. Reich, M.; Kesler, S.E.; Utsunomiya, S.; Palenik, C.S.; Chryssoulis, S.L.; Ewing, R. Solubility of gold in arsenian pyrite. Geochim. Cosmochim. Acta 2005, 69, 2781-2796. [CrossRef]

60. Rye, R.O.; Ohmoto, H. Sulphur and carbon isotopes and ore genesis: A review. Econ. Geol. 1974, 69, 826-842. [CrossRef]

61. Rollinson, H.R. Using Geochemical Data: Evaluation, Presentation, Interpretation; Longman: Singapore, 1996; pp. 1-314.

62. Ohmoto, H.; Rye, R.O. Isotopes of sulphur and carbon. In Geochemistry of Hydrothermal Ore Deposits; Barnes, H.L., Ed.; Wiley: New York, NY, USA, 1979; pp. 509-567.

63. Sangster, A.L. Light stable isotope evidence for a metamorphogenic orogen for bedding-parallel, gold-bearing veins in Cambrian flysch, Meguma Group. Explor. Min. Geol. 1992, 1, 69-79.

64. Goldfarb, R.J.; Miller, L.D.; Leach, D.L.; Snee, L.W. Gold deposits in metamorphic rocks of Alaska. Econ. Geol. 1997, 9, 151-190.

65. Phillips, G.N.; Groves, D.I.; Brown, I.J. Source requirements for the Golden Mile, Kalgoorie: Significance to the metamorphic replacement model for Archean gold deposits. Can. J. Earth Sci. 1987, 24, 1643-1651. [CrossRef]

66. Oppenheimer, C.; Fischer, T.B.; Scaillet, B. Volcanic degassing: Process and impact. In Treatise on Geochemistry, 2nd ed.; Heinrich, D.H., Turekian, K., Eds.; Elsevier: Amsterdam, The Netherlands, 2014; pp. 111-179.

67. Bowman, J.R. Stable-isotope systematics of skarns. In Mineralised Intrusion-Related Skarn Systems; Lentz, D.R., Ed.; Mineralogical Association of Canada: Ottawa, ON, Canada, 1998; Volume 26, pp. 59-414. 
68. Parnell, J.; Perez, M.; Armstrong, J.; Bullock, L.; Feldmann, J.; Boyce, A.J. A black shale protolith for gold-tellurium mineralisation in the Dalradian Supergroup (Neoproterozoic) of Britain and Ireland. Appl. Earth Sci. 2017, 126, 161-175. [CrossRef]

69. Craig, H. The geochemistry of the stable carbon isotopes. Geochim. Cosmochim. Acta 1953, 3, 53-92. [CrossRef]

70. Sackett, W.M. The depositional history and isotopic organic carbon composition of marine sediments. Mar. Geol. 1964, 2, 173-185. [CrossRef]

71. Degens, E.T. Biogeochemistry of stable carbon isotopes. In Organic Geochemistry Methods and Results; Eglinton, G., Murphy, M.T.J., Eds.; Springer: Berlin/Heidelberg, Germany, 1969; pp. 304-329.

72. Schidlowski, M.; Hayes, J.M.; Kaplan, I.R. Isotopic inferences of ancient biochemistries: Carbon, sulphur, hydrogen, and nitrogen. In Earth's Earliest Biosphere: Its Origin and Evolution; Schopf, J.W., Ed.; Princeton University Press: Princeton, NJ, USA, 1983; pp. 149-187. 